\title{
Childhood socioeconomic position and adult leisure-time physical activity: a systematic review
}

\author{
Ahmed Elhakeem ${ }^{*}$, Rachel Cooper ${ }^{1}$, David Bann² and Rebecca Hardy ${ }^{1}$
}

\begin{abstract}
Regular leisure-time physical activity (LTPA) benefits health and is thought to be less prevalent in lower socioeconomic groups. Evidence suggests that childhood socioeconomic circumstances can impact on adult health and behaviour however, it is unclear if this includes an influence on adult LTPA. This review tested the hypothesis that a lower childhood socioeconomic position (SEP) is associated with less frequent LTPA during adulthood. Studies were located through a systematic search of MEDLINE, Embase, PsycINFO, CINAHL and SPORTDiscus and by searching reference lists. Eligible studies were English-language publications testing the association between any indicator of childhood SEP and an LTPA outcome measured during adulthood. Forty-five papers from 36 studies, most of which were European, were included. In most samples, childhood SEP and LTPA were self-reported in midlife. Twenty-two studies found evidence to support the review's hypothesis and thirteen studies found no association. Accounting for own adult SEP partly attenuated associations. There was more evidence of an association in British compared with Scandinavian cohorts and in women compared with men. Results did not vary by childhood SEP indicator or age at assessment of LTPA. This review found evidence of an association between less advantaged childhood SEP and less frequent LTPA during adulthood. Understanding how associations vary by gender and place could provide insights into underlying pathways.
\end{abstract}

Keywords: Socioeconomic position, Socioeconomic inequalities, Physical activity, Exercise, Life course, Systematic review

\section{Introduction}

Physical activity (PA) is an important modifiable health behaviour implicated in the prevention of chronic disease and the promotion of health and mental well-being [1]. In addition, physical inactivity is a substantial public health burden [2]. Of the different domains of PA, leisure-time PA (LTPA) makes up the majority of time spent in moderate-to-vigorous intensity PA [3] and tends to be more strongly associated with favourable health outcomes [1, 4]. Evidence that LTPA levels have increased over time suggest that this domain of PA could be easier to modify than others [5] however, LTPA interventions generally report only small and short-term benefits [6].

Like many health-related outcomes, evidence from existing reviews indicates that LTPA is associated with contemporaneous socioeconomic circumstances [7-9]. Despite inconsistencies in the results as well as disagreement over whether certain indicators of socioeconomic position (SEP)

\footnotetext{
* Correspondence: ahmed.elhakeem.13@ucl.ac.uk

${ }^{1}$ MRC Unit for Lifelong Health and Ageing at UCL, 33 Bedford Place, London WC1B 5JU, UK

Full list of author information is available at the end of the article
}

end the article appear to be more strongly related to LTPA than others $[8,9]$, the evidence suggests that less socioeconomically advantaged youth [7] and adults $[8,9]$ tend to participate less frequently in LTPA compared with their more advantaged peers.

In addition to more temporally adjacent associations between SEP and health, considerable evidence links childhood socioeconomic circumstances to adult health and behavioural outcomes [10]. These associations are typically of substantial magnitude and are not fully explained by the continuity of socioeconomic circumstances from childhood into adulthood [10]. It is plausible that adult LTPA mediates some of these associations or that adult LTPA itself exhibits early life socioeconomic origins. However, studies of the association between childhood SEP and adult LTPA have been inconsistent [11] and the literature has not been systematically reviewed.

A systematic review was carried out to test the hypothesis that a lower childhood SEP is associated with less frequent LTPA during adulthood. The extent to which associations were explained by the continuity of SEP from

C Biomed Central

(C) 2015 Elhakeem et al. This is an Open Access article distributed under the terms of the Creative Commons Attribution License (http://creativecommons.org/licenses/by/4.0), which permits unrestricted use, distribution, and reproduction in any medium, provided the original work is properly credited. The Creative Commons Public Domain Dedication waiver (http:// creativecommons.org/publicdomain/zero/1.0/) applies to the data made available in this article, unless otherwise stated. 
childhood into adulthood and between-study heterogeneity were explored.

\section{Methods}

This systematic review, which was registered with the PROSPERO database (CRD42014007063) in January 2014, was carried out following the PRISMA guidelines [12] and a study protocol [13].

\section{Eligibility criteria}

Included studies were those that tested the association between any recalled or prospectively ascertained indicator of childhood SEP (up to age 18 years) and an LTPA outcome measured from age 25 . Studies were included if they reported results in English and published their findings in peer-reviewed journals. Observational studies using population-based samples were considered for inclusion.

Eligible indicators of childhood SEP were any resource and/or prestige-based measures of position within a societal structure [14] referring to participants' early life (e.g. parental occupation/education, household amenities). Participants' own education was not considered an eligible exposure despite its occasional use as an indicator of childhood SEP as it also captures the influence of adult resources [15].

Any PA performed during leisure-time was considered including sport, exercise and total LTPA [16]. The minimum age of 25 at measurement of LTPA, which equates the United Nations' definition of adulthood [17], allows us to examine the long-term influences of childhood SEP and to inspect, in studies that account for own adult SEP, whether any associations are explained by the continuity of SEP from childhood to adulthood.

Reviews, unpublished literature, studies with non-LTPA outcomes (e.g. occupational PA only) or non-community based samples (e.g. hospital inpatients) were excluded.

\section{Search strategy}

Embase (from 1974), MEDLINE (from 1946), PsycINFO (from 1806), CINAHL (from 1937) and SPORTDiscus (from 1985) were systematically searched using free-text synonym key-words (see Additional file 1) to locate all eligible studies available up to December 2014. Proximity and Boolean logic operators and truncation commands were used during the search [13]. Reference lists of included papers were searched to locate additional studies.

\section{Study selection}

Results of the database searches were merged and duplicates removed. Abstracts were screened by two researchers (from $\mathrm{AE}, \mathrm{RC}$ and $\mathrm{RH}$ ) working independently and remaining full-texts of potentially eligible papers were double screened for inclusion. Disagreements were resolved through discussion between $\mathrm{AE}, \mathrm{RC}$ and $\mathrm{RH}$.

\section{Data extraction}

The following data were extracted from all included papers (see Additional file 2): citation details, study details (e.g. design, setting, sample size), exposure and outcome details (e.g. type of indicators used and how and when these were ascertained), participant details (e.g. age, gender), statistical methods used, information on adjustment for potential confounding and mediating factors and lists of potentially eligible papers identified from reference lists. We extracted all statistics relating to the association of interest. A planned meta-analysis [13] was not attempted due to considerable heterogeneity in the reporting of results. All data were double extracted (by $\mathrm{AE}, \mathrm{RC}, \mathrm{DB}$ and $\mathrm{RH}$ ) and discrepancies were resolved through discussion between these authors.

\section{Quality assessment}

Study quality was assessed using the Newcastle-Ottawa Scale [18] which was modified [19] for this review. Quality was judged based on representativeness (of the study and source populations), adjustment for covariates, length of follow-up and methodology used to measure childhood SEP and adult LTPA (see Additional file 3). Quality scores were calculated as the average of two reviewers' ratings with a potential range from 0 (lowest quality) to 9 (highest quality).

\section{Results}

A total of 1782 citations were identified. After initial screening and full-text assessments, 45 papers [20-64] reporting findings from 36 study samples underwent data extraction and were included for review (Fig. 1).

Characteristics of the included papers are presented in Table 1. Most (34/45) were based on European samples including 18 UK papers reporting on ten different study populations and 11 Scandinavian papers each from a unique study sample (four from Finland, three from Denmark and two from each of Norway and Sweden). Two papers each (from 2 studies) from Belgium and the Netherlands and one from Spain complete the European study settings. The remaining papers were eight US, one Australian, and two Chinese papers (the latter both reporting findings from the Guangzhou Bio-bank study (GBCS)). Some included papers did not address the review's question as the primary association of interest and treated PA as a confounding or mediating factor but presented relevant associations [24, 27, 31, 36, 41, $52,56,57,61]$.

Study sample sizes varied from 112 to 20,086 and mostly comprised adults whose LTPA was ascertained in midlife. Birth years were from the early 1900 s to 1980 and participants were mostly drawn from the general population though four study populations were sampled from occupational settings [26-30, 41, 58]. The majority 


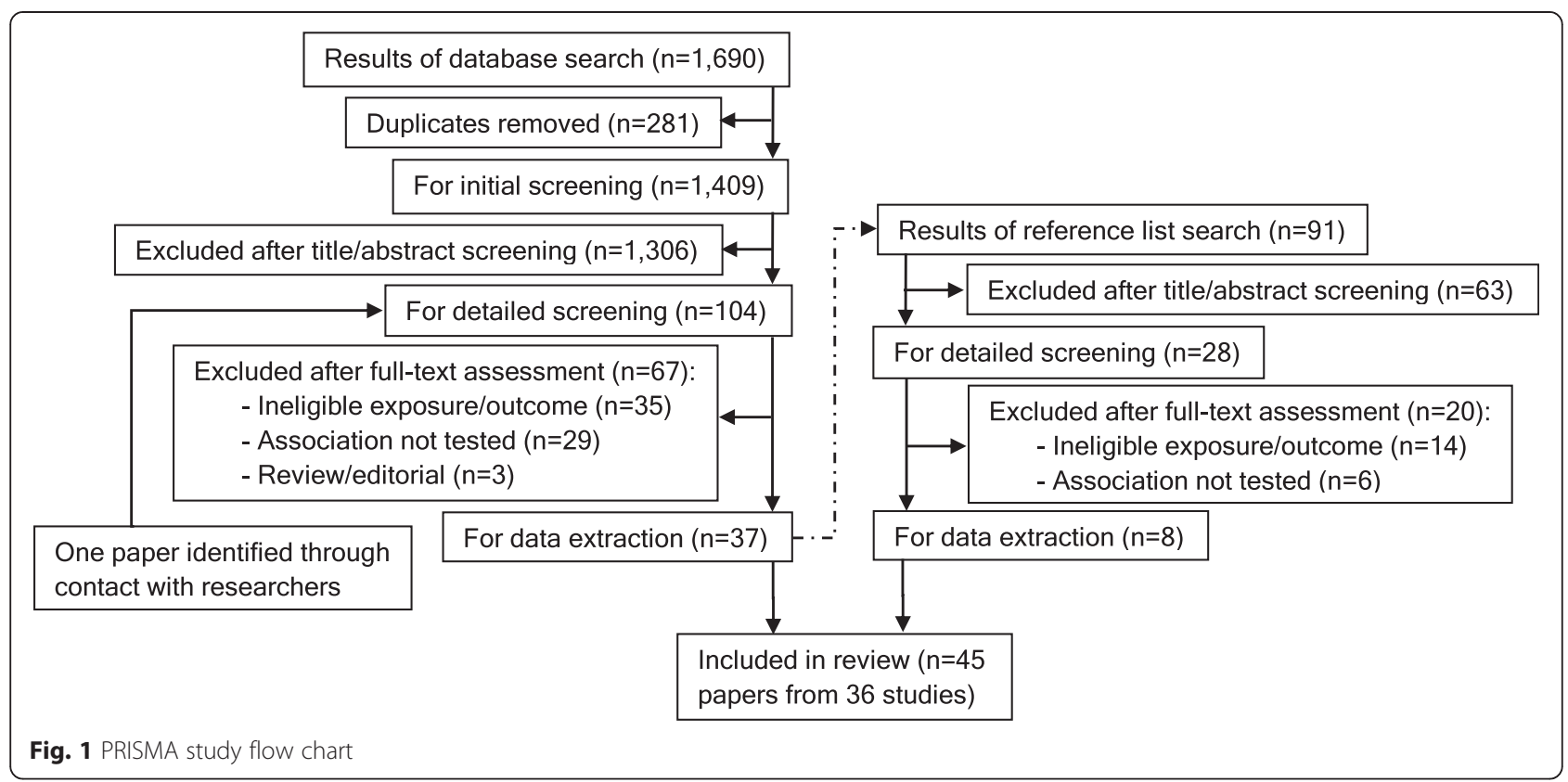

of included papers $(n=34)$ had a medium quality score (3 to 5 ) although the range was considerable (0.5 to 7).

Twenty-nine papers (22 studies) relied on participants recalling childhood SEP and in sixteen (14 studies) it was ascertained prospectively. For this review, different measures of childhood SEP were grouped into a) parental occupation, b) parental education and c) indices (combining $>1$ measure) and other indicators of childhood SEP (e.g. car access). Eight papers (7 studies) [20, 22, 23, $33,35,44,50,56]$ present results from at least two of the above and four (4 studies) $[20,35,44,50]$ report associations for each group of childhood SEP measures. PA was measured by self-report with the exception of Beunen et al. [50] who present both accelerometer and self-reported outcomes. Questions used to collect PA ranged from single-items $[40,44,59]$ to detailed questionnaires [34]. Not all outcomes were LTPA-specific as three papers present outcomes conflating work-related activity and LTPA $[33,55,56]$ and some provide no description of what PA domains are included in their outcome (but which are assumed to include LTPA) $[25,57,61]$.

\section{Association between childhood SEP and adult LTPA}

Results were presented as prevalence of LTPA by childhood SEP group, correlation between SEP and LTPA or regression coefficients from statistical models. Overall, results supported the hypothesis that a lower childhood SEP is associated with less frequent adult LTPA however, several null findings were reported. Two studies found evidence of an association between lower childhood SEP and higher adult PA [33, 62]. Results are summarised by three groups of childhood SEP indicators (Tables 2, 3, 4).

\section{Parental occupational class}

Thirty papers (22 studies) tested the association between parental occupation during childhood and adult LTPA and twenty-one (16 studies) found evidence that a lower parental occupational class was associated with less frequent LTPA during adulthood (Table 2). All UK studies used the Registrar General's Social Classification (RGSC) to categorise parental occupations into usually four or two groups. Studies from other countries used similar categorisations to those of the RGSC although several considered farming occupations as separate groups [43-45, 48, 49].

Evidence was available from three British birth cohorts initiated in 1946, 1958 and 1970. A higher prevalence of sports participation in higher paternal occupational groups was reported at age 36 in women from the MRC National Survey of Health and Development (NSHD) [34]. Later findings from this cohort [33] showed similar trends for LTPA derived by latent classes in both men and women and trends in the opposite direction for a combined walking during work and pleasure outcome (Table 2). Genderadjusted analyses from the next oldest cohort born in 1958, the National Child Development Study (NCDS), showed that a lower parental occupational class was associated with less LTPA at ages 33, 42 and 50 years [35]. This association was fully attenuated at age 33 after accounting for other early life factors and following further adjustments (including for own adult SEP), it was only seen at age 50 [35]. A second NCDS paper reported a non-significant correlation between parental occupation and exercise at age 50 [36]. Father's occupational class measured three times during early life was associated with LTPA at age 34 in the 1970 British cohort study [37]. 
Table 1 Characteristics of the included studies: arranged by region/country and from older to younger age at measurement of physical activity

\begin{tabular}{|c|c|}
\hline -1st author (year) & -Description \\
\hline -Country ${ }^{a}$ (birth year/s) & -Age at physical activity assessment \\
\hline -Study name & -Sample size (\% female) \\
\hline -Johnson (2011) [20] & -Scottish birth cohort. \\
\hline -UK (1936) & -70 years. \\
\hline -Lothian Birth Cohort 1936 & $-1091(49.8 \%)$ \\
\hline -Lawlor (2004) [21] & $\begin{array}{l}\text {-Cross-section of women recruited } \\
\text { from GP lists in } 23 \text { British towns. }\end{array}$ \\
\hline -UK (1921-40) & $-60-79$ years. \\
\hline $\begin{array}{l}\text {-British Women's Heart \& } \\
\text { Health Study (BWHHS) }\end{array}$ & $-3444(100 \%)$ \\
\hline -Hillsdon (2008) [22] & $\begin{array}{l}\text {-Cross-section of women recruited } \\
\text { from GP lists in } 23 \text { British towns. }\end{array}$ \\
\hline -UK (1921-40) & $-60-79$ years. \\
\hline -BWHHS & $-4103(100 \%)$ \\
\hline -Watt (2009) [23] & $\begin{array}{l}\text {-Cross-section of women recruited } \\
\text { from GP lists in } 23 \text { British towns. }\end{array}$ \\
\hline -UK (1921-40) & $-60-79$ years. \\
\hline -BWHHS & $-3523(100 \%)$ \\
\hline -Ramsay (2009) [24] & $\begin{array}{l}\text {-Cross-section of men recruited from } \\
\text { GP lists in } 24 \text { British towns. }\end{array}$ \\
\hline -UK (1920s-30s) & $-52-74$ years. \\
\hline -British Regional Heart Study (BRHS) & $-5188(0 \%)$ \\
\hline -Wannamethee (1996) [25] & $\begin{array}{l}\text {-Cross-section of men recruited from } \\
\text { GP lists in } 24 \text { British towns. }\end{array}$ \\
\hline -UK (1920s-30s) & $-40-59$ years. \\
\hline -BRHS & $-2188(0 \%)$ \\
\hline -Stringhini (2013) [26] & $\begin{array}{l}\text {-Cohort of civil servants employed } \\
\text { in London. }\end{array}$ \\
\hline -UK (1930-53) & $-40-59$ years (phase 3 ). \\
\hline -Whitehall II (WHII) Study & $-6387(28.5 \%)$ \\
\hline -Heraclides (2008) [27] & $\begin{array}{l}\text {-Cohort of civil servants employed } \\
\text { in London. }\end{array}$ \\
\hline -UK (1930-53) & $-44-69$ years (phase 5 ). \\
\hline -WHII Study & $-4598(26.8 \%)$ \\
\hline
\end{tabular}

-Childhood socioeconomic indicator $/ s^{\mathrm{b}} \quad$-Physical activity measurem

-Outcome/s of interest ${ }^{c}$

-How these were ascertained ${ }^{b}$

-Level of physical activities such as household

3.5

-PO (main occupation), $\mathrm{PE}, \mathrm{I} \& \mathrm{O}$

(number of people per room, shared toilet

chores, keep-fit, heavy exercise and sport.

facilities, whether indoor/outdoor toilet).

-Physical activity six point score.

-Recalled by SM at age 70 .

-PO (longest held occupation).

-Recalled by SM at age 60-79.

-Hours per week spent on several types of domestic, recreational and sports activities.

-Physically inactive $(<1 \mathrm{~h} /$ week. of moderate or vigorous physical activity).

PO (longest held occupation), I\&O (house with bathroom; hot water; shared bedroom, car access, and an index of all the above).

-Recalled by SM at age 60-79.

-PO (longest held occupation), I\&O (house with bathroom; hot water; shared bedroom, car access, and an index of all the above).

-Recalled by SM at age 60-79.

-PO (longest held occupation)

-Recalled by SM at age 52-74

-PO (longest held occupation)

-Recalled by SM at age 52-74.

PO (main occupation).

-Recalled by SM at age 35-55.

PO (main occupation).

-Recalled by SM at age 35-55

-Hours per week spent on several types of domestic, recreational and sports activities.

-Moderate to vigorous physical activity hours/week.

-Hours per week spent on several types of domestic, recreational and sports activities.

-Low exercise $(<2 \mathrm{~h} /$ week. of moderate or vigorous physical activity).

-Hours per week spent on several types of physical activities including walking, cycling and sports.

-Physically inactive (none or occasional physical activity).

-No description (reference provided).

-Physically active.

-Hours per week spent on moderate and vigorous physical activities.

-Physically inactive $(\leq 1 \mathrm{~h} /$ week. of moderate and $\leq 1 \mathrm{~h} /$ week. of vigorous physical activity).

-Hours per week spent on several types of domestic, recreational and sports activities.

-Sedentary lifestyle (low quintile of MET score). 
Table 1 Characteristics of the included studies: arranged by region/country and from older to younger age at measurement of physical activity (Continued)

-Brunner (1999) [28]

-UK (1930-53)

-WHII study

-Blane (1996) [29]

-UK (1908-37)

-West of Scotland Collaborative Study

-Hart (1998) [30]

-UK (1908-37)

-West of Scotland Collaborative Study

-Popham (2010) [31]

-UK (1949-68)

-2003 Scottish Health Survey

-Hart (2008) [32]

-UK (1937-66)

-Mid span family Study

-Silverwood (2012) [33]

-UK (1946)

-MRC National Survey of Health and Development (NSHD)

-Kuh \& Cooper (1992) [34]

-UK (1946)

-MRC NSHD

-Pinto Pereira (2014) [35]

-UK (1958)

-National Child Development Study 1958 (NCDS)

-Cheng \& Furnham (2013) [36] -UK (1958)

$-N C D S$
-Cohort of civil servants employed

in London.

$-35-55$ years (phase 1).

-6980 (31.6\%).

-Cross-section of men employed in 27 Scottish work places.

-35-64 years.

$-5645(0 \%)$.

-Cross-section of men employed in 27 Scottish work places.

$-35-64$ years.

-5567 (0 \%).

-Cross-section of Scottish residents.

$-35-54$ Years.

-2770 (\% unknown).

-Cross-section of the 1970s Renfrew/Paisley Study offspring.

$-30-59$ years.

$-2338(55.5 \%)$.

-British birth cohort.

$-36-53$ years.

$-3847(49.6 \%)$.

-British birth cohort.

-36 years.

-2144 (50.3\%).

-British birth cohort.

$-33,42,50$ years.

$-12,776 \mathrm{had} \geq$ one measure of LTPA.

-British birth cohort.

-50 years.

-5921 (49.4\%).
-PO (main occupation)

-Recalled by SM at age $35-55$.

-Hours per week spent on several types of domestic, recreational and sports activities.

-Physically inactive (no moderate or

vigorous activities).

-PO (main occupation).

-Recalled by SM at age 35-64.

-Hours per week spent on exercise outside work including walking, gardening and golfing.

-Exercise hours/week.

-Hours per week spent on exercise outside work including walking, gardening and golfing

-Recalled by SM at age 35-64.

-PO (when SM was aged 14)

-Recalled by SM at age 35-54

$-\mathrm{PO}$

-Reported by parents (SM was aged 6-39).

$-P O, P E$.

-Reported by parents (SM was aged 4 and 6).

$-P O, P E$.

-Reported by parents (SM was aged 4 and 6 ).

$-P O, P E, I \& O$ (index of household amenities: availability of bathroom, indoor lavatory and hot water).

-Reported by parents at SM's birth and when aged 7,11 and 16 .

-PO (current or last held occupation).

-Reported by parent at SM's birth
-Exercise hours/week.

-Frequency of several types sports and exercises during previous 4 weeks.

-Sport and exercise (participated $\geq$ once in sport/exercise at moderate/high intensity for $\geq 15 \mathrm{~min} /$ day).

-Frequency of daily activity and physical activity outside work.

-No exercise (not very/at all active in daily activities and active for < once/week. or never outside of work).

-Latent classes for a) walking during work and pleasure b) cycling during work and pleasure and c) LTPA.

-LTPA (low, gardening \& DIY, sports), walking, cycling (low, high)

-Frequency of several types of sports and recreational activities during previous month.

-High participation in sport and recreational activities.

-Frequency of LTPA such as swimming, going for walks.

-Low LTPA (< once/week).

-Frequency of physical exercise. 
Table 1 Characteristics of the included studies: arranged by region/country and from older to younger age at measurement of physical activity (Continued)

\begin{tabular}{|c|c|c|c|c|}
\hline -Juneau (2014) [37] & -British birth cohort. & $-\mathrm{PO}$ & -Frequency of LTPA during the previous 8 weeks. & 5 \\
\hline -UK (1970) & -34 years. & \multirow{2}{*}{$\begin{array}{l}\text {-Reported by parents at SM's birth and } \\
\text { when aged } 5 \text { and } 10 \text { years. }\end{array}$} & \multirow[t]{2}{*}{-Estimated LTPA energy expenditure. } & \\
\hline -1970 British Cohort Study & $-9624(52.2 \%)$ & & & \\
\hline -Osler (2008) [38] & $\begin{array}{l}\text {-Danish birth cohort of men from } \\
\text { Copenhagen. }\end{array}$ & $-\mathrm{PO}$ & $\begin{array}{l}\text {-Frequency of walking, running, cycling and } \\
\text { other activities. }\end{array}$ & 6.5 \\
\hline -Denmark (1953) & -51 years. & \multirow[t]{2}{*}{-Extracted from birth records. } & \multirow{2}{*}{$\begin{array}{l}\text {-Sedentary leisure activity (mainly reading, } \\
\text { watching TV or having other sedentary } \\
\text { activities during leisure). }\end{array}$} & \\
\hline -Metropolit Birth Cohort & $-6292(0 \%)$ & & & \\
\hline -Lynch (1997) [39] & $\begin{array}{l}\text {-Cross-section of men from Eastern } \\
\text { Finland. }\end{array}$ & \multirow{2}{*}{$\begin{array}{l}-1 \& O \text { (index of } \mathrm{PO}, \mathrm{PE} \text {, whether family } \\
\text { perceived as wealthy, whether family } \\
\text { lived on a farm and size of farm). }\end{array}$} & \multirow[t]{2}{*}{$\begin{array}{l}\text {-Energy expended in LTPA during the previous } \\
12 \text { months, e.g. jogging, swimming, cycling, skiing. }\end{array}$} & \multirow[t]{3}{*}{2.5} \\
\hline -Finland (1920s-40s) & $-42-60$ years. & & & \\
\hline $\begin{array}{l}\text {-Kuopio Ischaemic Heart Disease } \\
\text { Risk Factor Study }\end{array}$ & $-2682(0 \%)$ & -Recalled by SM at age $42-60$. & $\begin{array}{l}\text {-(i) No conditioning activities, (ii) low quartile } \\
\text { of conditioning activities. }\end{array}$ & \\
\hline -Kvaavik (2011) [40] & $\begin{array}{l}\text {-Follow-up of Oslo students invited } \\
\text { to a health education intervention. }\end{array}$ & $-P E$. & \multirow{2}{*}{$\begin{array}{l}\text {-'How often do you exercise for at least half an } \\
\text { hour to the extent that you sweat and/or are } \\
\text { short of breath?' }\end{array}$} & \multirow[t]{3}{*}{6} \\
\hline -Norway (1964-8) & $-25,33,40$ years. & \multirow[t]{2}{*}{-Reported by parents (SM aged 11-16). } & & \\
\hline -Oslo Youth Study & $-240,329,407$ & & -LTPA (twice/week). & \\
\hline -Jørgensen (2013) [41] & $\begin{array}{l}\text {-Cohort of Danish women employed } \\
\text { as social and health care assistants. }\end{array}$ & -PO (when SM was aged 14). & -Hours per week spent on LTPA. & 0.5 \\
\hline -Denmark $(\approx 1971)$ & -35.4 years $(S D=10.5)$ & \multirow[t]{2}{*}{-Recalled by SM at age 35.4 . } & \multirow[t]{2}{*}{ - Low LTPA (<4 h/week). } & \\
\hline -Danish Health Care Worker Cohort & $-1661(100 \%)$ & & & \\
\hline -Barnekow-Bergkvist (1998) [42] & -Follow-up of Swedish students. & $-\mathrm{PO}$ & \multirow{3}{*}{$\begin{array}{l}\text {-Hours per week spent on LTPA (includes sports, } \\
\text { walking, and cycling) in the previous } 12 \text { months. } \\
\text {-LTPA MET hours/week. }\end{array}$} & \multirow[t]{3}{*}{3} \\
\hline -Sweden (1958) & -34 years. & \multirow[t]{2}{*}{-Recalled by SM at age 34.} & & \\
\hline & $-278(43.5 \%)$ & & & \\
\hline -Tammelin (2003) [43] & -Northern Finland birth cohort. & $-\mathrm{PO}$ & -Frequency of light and brisk LTPA. & 5.5 \\
\hline -Finland (1966) & -31 years. & \multirow[t]{2}{*}{-Reported when SM aged 14.} & \multirow{2}{*}{$\begin{array}{l}\text {-Physically inactive (brisk LTPA < once/week. } \\
\text { and light LTPA <4 times/week). }\end{array}$} & \\
\hline -North Finland Birth Cohort 1966 & $-7794(53 \%)$ & & & \\
\hline -Makinen (2009) [44] & $\begin{array}{l}\text {-Regionally stratified cross-section of } \\
\text { Finnish adults. }\end{array}$ & \multirow{2}{*}{$\begin{array}{l}-\mathrm{PO}, \mathrm{PE}, \mathrm{I} \mathrm{OO} \text { (long-term financial problems } \\
\text { in family, regular parental unemployment- } \\
\text { both before age } 16 \text { ). }\end{array}$} & $\begin{array}{l}\text {-How much do you exercise and strain yourself } \\
\text { physically in leisure time?' }\end{array}$ & \multirow[t]{3}{*}{3.5} \\
\hline -Finland (1970 \& older) & $-30+$ years. & & \multirow{2}{*}{$\begin{array}{l}\text {-Inactive (read, watch TV or do other activities } \\
\text { that do not strain me physically); moderately } \\
\text { active (walk, cycle or move in other ways for } \\
\text { at least } 4 \mathrm{~h} / \text { week). }\end{array}$} & \\
\hline -Health 2000 Survey & $-7112(55.4 \%)$ & -Recalled by SM at age $30+$. & & \\
\hline -Wichstrøm (2013) [45] & $\begin{array}{l}\text {-Follow-up of students from } 67 \\
\text { Norwegian schools. }\end{array}$ & $-\mathrm{PO}$ & $\begin{array}{l}\text {-Hours spent on physical exercise during the } \\
\text { previous week. }\end{array}$ & 4.5 \\
\hline -Norway (1973-80) & $-25-32$ years. & \multirow[t]{2}{*}{-Reported by SM at age 12-19. } & \multirow[t]{2}{*}{-LTPA hours/week. } & \\
\hline -Young in Norway Study & $-2890-2923$. & & & \\
\hline
\end{tabular}


Table 1 Characteristics of the included studies: arranged by region/country and from older to younger age at measurement of physical activity (Continued)

\begin{tabular}{|c|c|c|}
\hline -Leino (1999) [46] & $\begin{array}{l}\text {-Follow-up of Finnish children } \\
\text { and adolescents. }\end{array}$ & $-P E$. \\
\hline -Finland (1962-71) & $-21-30$ years. & -Reported by SM at age 9-18. \\
\hline -Cardiovascular Risk in Young Finns Study & $-432(53.7 \%)$ & \\
\hline -Osler (2001) [47] & $\begin{array}{l}\text {-Follow-up of CCHS offspring } \\
\text { aged 6-18 at baseline. }\end{array}$ & $-P E$. \\
\hline -Denmark (1961-73) & $-19-31$ years. & -Reported by parents (SM aged 6-18). \\
\hline $\begin{array}{l}\text {-Offspring of Copenhagen City } \\
\text { Heart Study (CCHS) }\end{array}$ & $-317(48.9 \%)$ & \\
\hline -Peck (1994) [48] & -Cross-section of employed Swedes. & $-P O$ (during SM's childhood). \\
\hline \multirow[t]{2}{*}{-Sweden (1900s-60s) } & $-16-74$ years. & -Recalled by SM at age $16-74$. \\
\hline & $-12,695(50.4 \%)$ & \\
\hline -Regidor (2004) [49] & $\begin{array}{l}\text {-Cross-section of an older Spanish } \\
\text { population. }\end{array}$ & $-\mathrm{PO}$ \\
\hline \multirow[t]{2}{*}{-Spain (1940 \& older) } & $-60+$ years. & -Recalled by SM at age $60+$. \\
\hline & $-3658(54.6 \%)$ & \\
\hline -Beunen (2004) [50] & $\begin{array}{l}\text {-27-year follow-up of Flemish } \\
\text { speaking adolescent Belgian boys. }\end{array}$ & -PO, PE, I\&O (degree of urbanisation). \\
\hline -Belgium (1956) & -40 years. & -Reported by SM at age 14-18. \\
\hline $\begin{array}{l}\text {-Leuven Longitudinal Study of } \\
\text { Flemish Boys }\end{array}$ & $-166(0 \%)$ & \\
\hline -Scheerder (2006) [51] & $\begin{array}{l}\text {-20-year follow up of Flemish speaking } \\
\text { adolescent Belgian girls. }\end{array}$ & $-I \& O$ (index of $\mathrm{PO}$ and $\mathrm{PE})$. \\
\hline -Belgium (1961-7) & $-32-41$ years. & -Reported by SM at age 12-18. \\
\hline $\begin{array}{l}\text {-Leuven Longitudinal Study of } \\
\text { Flemish Girls }\end{array}$ & $-257(100 \%)$ & \\
\hline -Kamphuis (2013) [52] & $\begin{array}{l}\text {-Cross-section of men living in or } \\
\text { near Eindhoven. }\end{array}$ & -PO (when SM was aged 12). \\
\hline -Netherlands (1916-51) & $-40-75$ years. & -Recalled by SM at age $40-75$. \\
\hline -GLOBE Study & $-4894(0 \%)$ & \\
\hline -van de Mheen (1998) [53] & $\begin{array}{l}\text {-Cross-section of adults living in or } \\
\text { near Eindhoven. }\end{array}$ & -PO (when SM was aged 11). \\
\hline -Netherlands (1910s-60s) & $-25-74$ years. & -Recalled by SM at age $25-74$. \\
\hline $\begin{array}{l}\text {-Longitudinal Study on Socio-Economic } \\
\text { Health Differences }\end{array}$ & $-13,854$ (\% unknown). & \\
\hline
\end{tabular}

-Frequency and duration of exercise used to

form an LTPA index.

-Physically inactive $(\leq 25$ th percentile of LTPA

index, range $=0-52$ ).

-Current level of participation in LTPA and whether active in sports.

-Low LTPA (mostly sitting or light activity for $\geq 4 \mathrm{~h} /$ week. and not active in sports).

-Regular LTPA (no description).

-No regular LTPA.

-Type of physical activity done in spare time or at any time if retired/unemployed.

-Physically inactive (only report sedentary activities e.g. reading, watching TV).

-Frequency of sports, other leisure-time activities and accelerometer counts of daily physical activity.

-Sport, leisure-time, \& counts indices.

-Hours per week spent on sports during the previous year.

-Level of sports participation (hours/week./year)

-Hours per week spent on transport, leisure-time and sports related activities.

-Physically active ( $\geq 3.5 \mathrm{~h} /$ week. of sports and transport or leisure-time physical activity).

-Leisure-time physical exercise (no description).

-Frequent LTPA, and no LTPA. 
Table 1 Characteristics of the included studies: arranged by region/country and from older to younger age at measurement of physical activity (Continued)

\section{-Pudrovska (2013) [54]}

-US (1939-40)

-Wisconsin Longitudinal Study

-Wray (2005) [55]

-US (1941 \& older).

-Health \& Retirement Study (HRS);

Study of Asset \& Health

Dynamics (AHEAD)

-Bowen (2010) [56]

-US (1941 \& older)

-HRS merged with AHEAD and

two other cohorts

-Carroll (2011) [57]

-US (1950s-70s)

-Vaccination Immunity Project

-Frank (2003) [58]

-US (1930-50)

-Women Physician Health Study

-Tsenkova (2014) [59]

-US (1921-70)

-Midlife in the US Study

-Kern (2010) [60]

-US (1910s)

-Terman Life Cycle Study

-Phillips (2009) [61]

-US (1940s-70s)

-Adult Health and Behaviour Project
-Long-term follow-up of high schoo

graduates from Wisconsin.

-65 years.

-5778 (54.7\%).

-Follow-up of middle aged and

older US adults.

-51-61 years (HRS); 70+ years (AHEAD). $\quad$-Recalled by SM at age 51-61 (HRS)

-HRS: 6106 (57\%); AHEAD: 3636 (63\%).

-Cohort of middle aged and older US adults.

$-51+$ years.

$-18,465$ (60\%).

-Cross-section of Pennsylvanian adults recruited to a Hepatitis B vaccination project.

$-40-60$ years

$-153(59.8 \%)$.

-Cross-section of women physicians born in the US.

$-30-70$ years.

$-2884(100 \%)$

-Cross-section of US adults who participated in a biomarkers study.

$-25-74$ years.

-895 (54.6\%)

-Long-term follow-up of Californian children with high $\mathrm{IQ}$.

-25-61 years.

$-1114(50 \%)$.

-Cross-section of Pennsylvanian adults without serious illnesses.

$-30-54$ years.

$-811(51.4 \%)$
$-1 \& \mathrm{O}$ (index of $\mathrm{PO}, \mathrm{PE}$, family income,

father's occupational income and father's

occupational education).

-Reported when SM was aged 17-18.

-PE.

and $70+(A H E A D)$.

$-\mathrm{PO}$ (main occupation), PE

-Recalled by SM at age 51+.

$-1 \& O$ (index for every 2 years of childhood: whether parents owned home, number of a) bathrooms, b) people living in the home and c) vehicles owned)

-Recalled by SM at age 40-60.

-PE.

-Recalled by SM at age 30-70.

$-1 \& O$ (index of $\mathrm{PE}$, childhood welfare status and financial level growing up).

-Recalled by SM at age 25-74.

$-1 \& \mathrm{O}$ (index of PO and PE).

-Reported by parents (SM was aged 11).

-PE.

-Recalled by SM at age 30-54.
-Hours per month spent on light (e.g. walking, gardening, golfing) and vigorous (e.g. aerobics, jogging, swimming) physical activities.

-Physical activity index.

-Whether or not SM is a vigorous exerciser. Includes heavy housework, cycling, aerobics, running, jogging, swimming and physical labour at work.

-Low physical activity (not exercising $\geq 3$ times/week)

-Whether or not SM is a vigorous exerciser. Includes heavy housework, cycling, aerobics, running, jogging, swimming and physical labour at work.

-Vigorous exercisers ( $\geq 3$ times/week).

-Paffenbarger physical activity questionnaire (no description).

-Physical activity kilocalories expended per week.

-Exercise (no description).

-Exercising $\geq 30$ min on 3 times per week.

-'How often do you engage in vigorous physical activity long enough to work up a sweat (e.g. running/heavy lifting)?

-Exercise sessions per month.

-Avocational activities and hobbies including sport, gardening, music, art, writing, photography.

-Average physical activity METs.

-Paffenbarger physical activity questionnaire (no description).

-Physical activity kilocalories expended per week. 
Table 1 Characteristics of the included studies: arranged by region/country and from older to younger age at measurement of physical activity (Continued)

-Schooling (2007) [62]

-China (1955 \& older)

-Guangzhou Bio-bank

Cohort Study (GBCS)

-Elwell-Sutton (2011) [63]

-China (1955 \& older)

$-G B C S$

-Gall (2010) [64]

-Australia (1970s)

-Childhood Determinants of

Adult Health Study
-Cross-section of Guangzhou community club members.

\section{$-50+$ years.}

-9748 (71.9\%).

-Cross-section of Guangzhou community club members

$-50+$ years.

$-20,086(73.2 \%)$

-20-year follow-up of the Australian Schools Health \& Fitness Survey.

-26-36 years.

$-1973(52.8 \%)$.
$-1 \& O$ (number of parental possessions from $\quad-I P A Q$ used (no description).

a watch sewing machine and bicycle

during SM's childhood).

-Recalled by SM at age $50+$

$-1 \& 0$ (number of parental possessions from a watch, sewing machine and bicycle

during SM's childhood).

-Recalled by SM at age 50+

-PE.

-Recalled by SM at age 26-36
-Inactive, minimally active, and HEPA (vigorous activity $\geq 3$ days/week. at $\geq 1500$ MET minutes/ week, or activity 7 days/week. at $\geq 3000$ MET minutes/week).

-IPAQ used (no description).

-Inactive, minimally active, and HEPA (vigorous activity $\geq 3$ days/week. at $\geq 1500$ MET minutes/ week, or activity 7 days/week. at $\geq 3000$ MET minutes/week).

-Whether or not SM participated in $\geq 3 \mathrm{~h}$ of moderate/vigorous LTPA per week.

-LTPA ( $\geq 3 \mathrm{~h} /$ week)

\section{a UK United Kingdom, US United States, Nordic group of countries (Norway, Sweden, Finland and Denmark) considered as one region}

${ }^{\mathrm{b}} P O$ Parental occupation (usually based on father's occupation, more detail can be found in brackets if provided in the paper), $P E$ Parental education (years and/or level), I\&O Indices and other measures of childhood socioeconomic position (SEP), includes (i) indices combining different indicators of childhood SEP and (ii) single measures which are distinct from parental occupation and education, SM Study member

CLTPA Leisure-time Physical Activity, METS Metabolic equivalents, IPAQ International Physical activity Questionnaire, HEPA Health enhancing physical activity: acronym used in the two GBCS papers [62, 63] ${ }^{\mathrm{d}} Q A$ score Quality assessment score (average of two assessor's scores possible values are $0-9$ ) 
Table 2 Results of studies testing the association between parental occupational class and leisure-time physical activity (LTPA) in adults

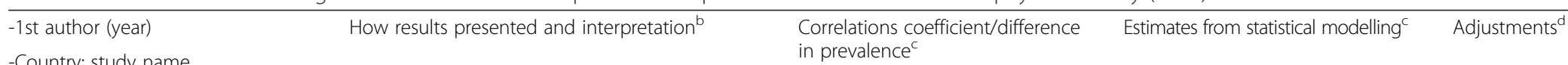

-Country; study name

-Sample size ; age

-Johnson (2011) [20]

Correlation and regression coefficients for

a 6-point LTPA score and parental

occupation (RGSC 1951: I, II, IIIN, IIIM, IV, V)

(per unit change from high to low

occupational class in regression model).

Prevalence of physical inactivity in six

parental occupational groups (RGSC
1980: I, II, IIIN, IIIM, IV, V) and odds of

physical inactivity per unit increase from

high to low occupational class.

$r=-0.06(+, p=0.05)$

-Lawlor (2004) [21]

-UK; British Women's Heart \&

Health Study (BWHHS)

$-3444^{\circ} ; 60-79$ years.

-Hillsdon (2008) [22]

-UK; BWHHS

$-4103^{\circ} ; 60-79$ years.

-Watt (2009) [23]

$-U K$; BWHHS

$-3523^{\circ} ; 60-79$ years.

-Ramsay (2009) [24]

-UK; British Regional Heart

Study (BRHS)

$-5188^{\hat{\alpha}} ; 52-73$ years.

-Wannamethee (1996) [25]

-UK; BRHS

$-5516^{\circledR} ; 40-59$ years.

-Stringhini (2013) [26]

-UK; Whitehall II (WHII) Study

$-6387 ; 40-59$ years.

-Heraclides (2008) [27]

-UK; WHII Study

-4598; 44-69 years.

Prevalence of manual parental occupational class (RGSC 1980) in four groups of physical activity hours/week.

Percentage difference in low exercise between manual (M) and non-manual (NM) parental occupations (RGSC 1980).

Prevalence of physical inactivity in manual (M) and non-manual (NM) parental occupations (RGSC 1980).

Prevalence of physical activity in manual (M) and non-manual (NM) parental occupations (RGSC 1980).

$(+, p<0.01)$

$\mathrm{NM}-\mathrm{M}=2.4 \%$ (ns)

Odds of physical inactivity in the lowest

occupation (RGSC 1980).

NM-M:

$\widehat{\delta}=1.9 \%$ (ns)

우 $=1.3 \%$ (ns) and non-manual (NM) parental occupations (RGSC 1980).
$\mathrm{I}-\mathrm{V}=-11.4 \%\{-13.6 ;-6.4\}(+)$

$$
\beta=-0.01 \text { (ns) }
$$
None

Education, own

occupational

Class, other childhood

SEP, IQ \& more

None

$\mathrm{OR}=1.17\{1.08 ; 1.26\}(+)$

$\mathrm{OR}=1.15\{1.06 ; 1.25\}(+)$

$\%$ manual occupations:

$\geq 3-0 \mathrm{~h} /$ week. $=-7.4 \%$

$\{-6.1 ;-8.6\}(+, p<0.001)$

$\mathrm{NM}-\mathrm{M}=-6.7 \%\{-2.5 ;-10.9\}$

None

$\mathrm{NM}-\mathrm{M}=-48 \%(+, p=0.05)$

None

$\mathrm{NM}-\mathrm{M}=8 \%(+, p<0.0001)$

None

Age, own occupational

class

compared to the highest tertile of parental

$\mathrm{OR}=1.37\{1.14 ; 1.65\}(+, p<0.05)$

Age, sex, ethnicity,

CHD, stroke, cancer,

hypertension, family

history of diabetes

None 
Table 2 Results of studies testing the association between parental occupational class and leisure-time physical activity (LTPA) in adults (Continued)

\begin{tabular}{|c|c|c|c|c|}
\hline -Brunner (1999) [28] & \multirow{4}{*}{$\begin{array}{l}\text { Prevalence of physical inactivity in four } \\
\text { parental occupational groups (RGSC 1980: } \\
\text { I/II, IIIN, IIIM, IVN). }\end{array}$} & \multicolumn{2}{|l|}{$\mathrm{I}-\mathrm{IV}\left(\mathrm{o}^{\Uparrow}\right)=-4.8 \%(+, p=0.01)$} & \multirow[t]{2}{*}{ Age } \\
\hline -UK; WHII Study & & \multicolumn{2}{|l|}{$\mathrm{I}-\mathrm{IV}(q)=-7.9 \%(+, p=0.02)$} & \\
\hline \multirow[t]{2}{*}{$-6980 ; 35-55$ years. } & & \multicolumn{2}{|l|}{$\mathrm{I}-\mathrm{IV}(\widehat{\jmath})=-2.6 \%$ (ns) } & \multirow{2}{*}{$\begin{array}{l}\text { Age, own occupational } \\
\text { class }\end{array}$} \\
\hline & & \multicolumn{2}{|l|}{$\mathrm{I}-\mathrm{IV}($ ( $)=-2.9 \%$ (ns) } & \\
\hline -Blane (1996) [29] & \multirow{3}{*}{$\begin{array}{l}\text { Prevalence and regression coefficients for } \\
\text { mean exercise hours/week. by four parental } \\
\text { occupational groups (RGSC 1966: I/II, IIIN, } \\
\text { IIIM, IVN). }\end{array}$} & \multirow{3}{*}{$\begin{array}{l}\text { I/II-IV } N=0.7 \mathrm{~h} / \text { week. } \\
\{\mathrm{SE}: \mathrm{I} / \mathrm{II}=0.13 ; \mathrm{IV} N=0.16\}\end{array}$} & & \multirow[t]{2}{*}{ Age } \\
\hline $\begin{array}{l}\text {-UK; West of Scotland } \\
\text { Collaborative Study }\end{array}$ & & & & \\
\hline$-5646^{\star} ; 35-64$ years. & & & $\beta=-0.16\{-0.32 ; 0.01\}$ (ns) & Age \\
\hline -Hart (1998) [30] & \multirow{3}{*}{$\begin{array}{l}\text { Prevalence of exercise hours/week. in four } \\
\text { groups of parental and own occupations } \\
\text { (RGSC 1966: 1. stable non-manual 2. moved } \\
\text { up 3. moved down 4. stable manual). }\end{array}$} & \multirow[t]{3}{*}{$1-4=0.5$ h/week. $(+, p=0.002)$} & & \multirow[t]{3}{*}{ Age } \\
\hline $\begin{array}{l}\text {-UK; West of Scotland } \\
\text { Collaborative Study }\end{array}$ & & & & \\
\hline$-5567^{3} ; 35-64$ years. & & & & \\
\hline -Popham (2010) [31] & \multirow{3}{*}{$\begin{array}{l}\text { Prevalence of sport \& exercise in four parental } \\
\text { occupational groups (RGSC: I/II, IIIN, IIIM, IVN). }\end{array}$} & \multirow[t]{3}{*}{ I/II-IVN = $18.6 \%\{17.7 ; 19.6\}(+)$} & & \multirow[t]{3}{*}{ Age, sex } \\
\hline -UK; 2003 Scottish Health Survey & & & & \\
\hline$-2770 ; 35-54$ years. & & & & \\
\hline -Hart (2008) [32] & \multirow{5}{*}{$\begin{array}{l}\text { Prevalence of no exercise in manual (M) and } \\
\text { non-manual (NM) parental occupations } \\
\text { (RGSC 1966) and odds of no exercise per } \\
\text { unit increase (1-6) from low to high parental } \\
\text { occupational class. }\end{array}$} & \multirow{2}{*}{\multicolumn{2}{|c|}{$\begin{array}{l}\text { NM-M: } \\
\widehat{\jmath}=3.7 \% \text { (ns) }\end{array}$}} & \multirow[t]{2}{*}{ None } \\
\hline -UK; Mid span Family Study & & & & \\
\hline \multirow[t]{3}{*}{$-2338 ; 30-59$ years. } & & \multirow[t]{3}{*}{$q=-3.0 \%(\mathrm{~ns})$} & Odds Ratios: & \multirow[t]{3}{*}{ Age } \\
\hline & & & $\hat{\jmath}=1.03(0.91 ; 1.16)(\mathrm{ns})$ & \\
\hline & & & $q=1.09(0.98 ; 1.21)(\mathrm{ns})$ & \\
\hline -Silverwood (2012) [33] & \multirow{6}{*}{$\begin{array}{l}\text { Prevalence of LTPA (low; gardening; sport \& } \\
\text { leisure), walking and cycling during work \& } \\
\text { for pleasure (high, low) in four parental } \\
\text { occupational groups (RGSC 1970: I/III, IIIN, } \\
\text { IIIM, IVN). }\end{array}$} & \multicolumn{2}{|l|}{ I/II-IVN: LTPA (sports \& leisure): } & \multirow[t]{6}{*}{ None } \\
\hline \multirow{2}{*}{$\begin{array}{l}\text {-UK; MRC National Survey of Health } \\
\text { and Development (NSHD) }\end{array}$} & & \multicolumn{2}{|l|}{$\hat{\sigma}=12.2 \%(+, p<0.001)$} & \\
\hline & & \multicolumn{2}{|l|}{$q=17.9 \%(+, p<0.001)$} & \\
\hline \multirow[t]{3}{*}{-> 3300; 31-53 years. } & & \multicolumn{2}{|l|}{ I/II-IVN: Walking (high): } & \\
\hline & & \multicolumn{2}{|l|}{$\hat{\sigma}=-17.6 \%(-, p<0.001)$} & \\
\hline & & \multicolumn{2}{|l|}{$q=-6.6 \%(-, p=0.002)$} & \\
\hline -Kuh \& Cooper (1992) [34] & Prevalence of most active in sports \& & I/II-IVN: & & None \\
\hline -UK; MRC NSHD & occupational groups (RGSC 1970: & $\hat{\delta}=9.1 \%(n s)$ & & \\
\hline$-2977 ; 36$ years. & & $q=21.4 \%(+, p<0.001)$ & & \\
\hline
\end{tabular}


Table 2 Results of studies testing the association between parental occupational class and leisure-time physical activity (LTPA) in adults (Continued)

-Pinto Pereira (2014) [35] Odds of low LTPA per unit increase from

-UK. National Child Development high to low parental occupational class Study 1958 (NCDS)

$-12,776$ had $\geq$ one measure of

LTPA; 33, 42, 50 year.

-Cheng \& Furnham (2013) [36]

$-U K ;$ (NCDS)

$-5921 ; 50$ year.

-Juneau (2014) [37]

-UK; 1970 British Cohort Study

-9624; 34 years.
(RGSC 1951: I/II, IIIN, IIIM, IVN). and parental occupation (RGSC 1951: 1,

IIINM, IIIM, IV, V) with higher scores for

higher occupational classes.

Correlation between LTPA (0-224 with

for lower occupational classes.
Age $42=1.10\{1.05 ; 1.15\}(+)$

23 unique values) and parental occupation

(RGSC: I, II, IIIN, IIIM, IVN) with higher scores

今: $r=-0.080(+, p<0.001)$

o: $r=-0.053(+, p<0.001)$

\section{Odds Ratios:}

Age $33=1.12\{1.07 ; 1.16\}(+)$

Age $42=1.16\{1.11 ; 1.20\}(+)$

Age $50=1.23\{1.17 ; 1.29\}(+)$

Age $33=1.06\{1.01 ; 1.11\}(+)$

Age $50=1.13\{1.07 ; 1.19\}(+)$

Age $33=1.01\{0.97 ; 1.06\}$ (ns)

Age $42=1.05\{1.002 ; 1.10\}(+)$

Age $50=1.09\{1.03 ; 1.15\}(+)$

Age $33=1.00(0.95 ; 1.05)$ (ns)

Age $42=1.04$ (0.99; 1.09) (ns)

Age $50=1.07(1.01 ; 1.13)(+)$

$r=-0.020$ (ns)

None

Sex

Sex, parental education aptitude, household amenities, cognition, lifestyle factors age 16, \& more

As above plus own

education, own socia

class, BMI, mental

health, number of

children in the

household, limiting

illness

None

Age 0

None

Age 5

入: $r=-0.048(+, p<0.001)$

o : $r=-0.077(+, p<0.001)$

Age 10

đ: $r=-0.086(+, p<0.001)$

o: $r=-0.064(+, p<0.001)$ 
Table 2 Results of studies testing the association between parental occupational class and leisure-time physical activity (LTPA) in adults (Continued)

\begin{tabular}{|c|c|c|c|c|}
\hline & \multicolumn{2}{|l|}{$\begin{array}{l}\text { Parameter estimates from structural equation } \\
\text { model (zero-inflated Poisson models) for LTPA } \\
\text { by parental occupation at birth and ages } \\
5 \text { and } 10 .\end{array}$} & $\begin{array}{l}\text { Parental occupation at birth: } \\
\text { Logistic portion of model: } \\
\hat{\delta}=0.054 \text { (ns) }\end{array}$ & \multirow[t]{2}{*}{$\begin{array}{l}\text { Occupational physical } \\
\text { activity, transport-related } \\
\text { physical activity }\end{array}$} \\
\hline & \multicolumn{2}{|l|}{$\begin{array}{l}\text { (Results presented from an accumulation of } \\
\text { risk with additive effects model (best fit), for } \\
\text { results for ages } 5 \text { and } 10 \text { see paper. }\end{array}$} & $\begin{array}{l}q=0.88(p<0.05) \\
\text { Counts portion of model: } \\
\hat{o}=-0.049(p<0.05) \\
q=0.050(p<0.05)\end{array}$ & \\
\hline -Osler (2008) [38] & \multirow{2}{*}{\multicolumn{2}{|c|}{$\begin{array}{l}\text { Odds of sedentary leisure activity in low } \\
\text { compared to high parental occupational class. }\end{array}$}} & $\mathrm{OR}=1.10\{0.97 ; 1.26\}$ & Age \\
\hline $\begin{array}{l}\text {-Denmark; } 1953 \text { Metropolit } \\
\text { Birth Cohort } \\
-6292^{\circledR} ; 51 \text { year. }\end{array}$ & & & $\mathrm{OR}=0.90\{0.78 ; 1.05\}$ & $\begin{array}{l}\text { Age, own education, own } \\
\text { occupational class, divorce, } \\
\text { cognition }\end{array}$ \\
\hline $\begin{array}{l}\text {-Jørgensen (2013) [41] } \\
\text {-Denmark; Danish Health Care } \\
\text { Worker Cohort } \\
-1661^{\circ} ; 35.4 \text { years (mean) }\end{array}$ & $\begin{array}{l}\text { Prevalence of low LTPA in five parental } \\
\text { occupational groups ( } 1 \text {. higher professional } \\
\text { 2. lower professional/non-routine M } \\
\text { 3. self-employed 4. skilled blue-collar } \\
\text { 5. unskilled blue-collar) }\end{array}$ & $\begin{array}{l}1-5: \\
q=-5.7 \%(+, p=0.011)\end{array}$ & & None \\
\hline $\begin{array}{l}\text {-Barnekow-Bergkvist (1998) [42] } \\
\text {-Sweden } \\
\text {-278; } 34 \text { years. }\end{array}$ & \multicolumn{2}{|l|}{$\begin{array}{l}\text { Regression coefficients for LTPA MET } \\
\text { hours/week. comparing non-manual to } \\
\text { manual parental occupations. }\end{array}$} & $\begin{array}{l}\beta: \\
\widehat{\sigma}=\text { reported as ns } \\
q=0.18(+)\end{array}$ & $\begin{array}{l}\text { Own education, sport club } \\
\text { member, two-hand lift, } \\
\text { attitudes to soccer \& } \\
\text { handball }\end{array}$ \\
\hline $\begin{array}{l}\text {-Tammelin (2003) [43] } \\
\text {-Finland; } 1966 \text { North Finland } \\
\text { Birth Cohort } \\
\text {-7794; } 31 \text { year. }\end{array}$ & \multicolumn{2}{|l|}{$\begin{array}{l}\text { Odds of physical inactivity in parental } \\
\text { occupational groups ( } 1 \text {. skilled professional } \\
\text { 2. skilled worker 3. unskilled worker } \\
\text { 4. farmer) with skilled professional used as } \\
\text { reference category. }\end{array}$} & $\begin{array}{l}\text { Odds Ratios (4 vs. 1): } \\
\begin{array}{l}\hat{\delta}=1.18\{0.94 ; 1.49\} \text { (ns) } \\
q=0.80\{0.63 ; 1.02\} \text { (ns) }\end{array}\end{array}$ & After-school sports \\
\hline $\begin{array}{l}\text {-Makinen (2009) [44] } \\
\text {-Finland; Health } 2000 \text { Survey } \\
-6262 ; 30+\text { Yrs. }\end{array}$ & \multicolumn{2}{|l|}{$\begin{array}{l}\text { Odds of inactivity and moderate LTPA relative } \\
\text { to high LTPA in father's occupational groups } \\
\text { (office employee, manual worker, self-employed, } \\
\text { farmer) with office employee used a reference } \\
\text { category. }\end{array}$} & $\begin{array}{l}\text { Inactivity }\left({ }^{\Uparrow}\right)=1.69(+) \\
\text { Inactivity }(+)=0.97 \text { (ns) } \\
\text { Moderate LTPA }\left({ }^{\Uparrow}\right)=1.68 \text { (ns) } \\
\text { Moderate LTPA }(+)=1.08 \text { (ns) }\end{array}$ & Age \\
\hline$-3905 ; 30+$ Yrs. & \multicolumn{2}{|l|}{$\begin{array}{l}\text { Odds of inactivity and moderate LTPA relative } \\
\text { to high LTPA in mother's occupational groups } \\
\text { (office employee, manual worker, self-employed, } \\
\text { farmer) with office employee used a reference } \\
\text { category. }\end{array}$} & $\begin{array}{l}\text { ORs (farmer vs. office employee): } \\
\text { Inactivity }\left({ }^{\Uparrow}\right)=1.49 \text { (ns) } \\
\text { Inactivity }(+)=0.87 \text { (ns) } \\
\text { Moderate LTPA }\left({ }^{\Uparrow}\right)=1.99 \text { (ns) } \\
\text { Moderate LTPA }(+)=1.40(+)\end{array}$ & Age \\
\hline
\end{tabular}

Odds of inactivity and moderate LTPA relative (office employee, manual worker, self-employed, carmer) with office employee used a reference

Odds of inactivity and moderate LTPA relative ( farmer) with office employee used a reference

Moderate LTPA $($ P $)=1.40(+)$ 
Table 2 Results of studies testing the association between parental occupational class and leisure-time physical activity (LTPA) in adults (Continued)

-Wichstrøm (2013) [45]

-Norway

-> 2800; 25-32 years

-Peck (1994) [48]

-Sweden

-13,695; 16-74 years.

-Beunen (2004) [50]

-Belgium; Leuven Longitudinal

Study of Flemish Boys

$-166^{\hat{2}} ; 40$ year.

-Kamphuis (2013) [52]

-Netherlands; GLOBE Study

$-4894^{\star} ; 40-75$ years.

-van de Mheen (1998) [53]

-Netherlands; Longitudinal Study on Socio-Economic Health

Differences

$-13,854 ; 25-74$ years.

-Regidor (2004) [49]

-Spain

$-3658 ; 60+$ Yrs.
LTPA in five parental occupational groups (leader, high professional, low professional, manual, farmer/fisherman).

Risk of no regular physical activity compared to the sample average in seven parental occupational groups (self-employed with employees, self-employed w/o employees, higher non-manual, assistant non-manual, skilled manual, unskilled manual, farmers).

Correlation and regression coefficients for sport, leisure-time and counts indices by parental occupation. Only leisure-time presented in paper

Prevalence of inactive, little and moderately active in three parental occupational groups (1. professional 2. white collar 3. blue collar).

Odds of no LTPA and frequent LTPA by parental occupation (1. higher grade professional 2. lower grade professional/ professional 2. lower grade professional/
routine NM 3. self-employed 4. high/low skilled M 5. unskilled M) with higher grade professional used a reference category.

Prevalence and odds of physical inactivity in four parental occupational groups (1. professional, manager, proprietor, clerical worker 2. self-employed farmer

3. skilled/unskilled manual worker

4. paid farm worker) with professional group used as reference category.

Odds of physical inactivity in manual compared to non-manual parental occupations.

Reported as 'unrelated to

LTPA at any time point' (ns)

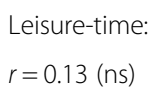

Leisure-time:

$r=0.13$ (ns)

1-3:

Inactive $=1.5 \%$ (ns)

Little active $=-0.9 \%$ (ns)

Moderately active $=2 \%$ (ns)

$1-4\left({ }^{\top}\right)=-9.5 \%(+, p=0.043)$ $1-4(q)=-7.9 \%(+, p=0.011)$
Unskilled manual:

$\hat{o}=1.24$ (ns)

q $=1.24$ (ns)

Higher non-manual:

$\widehat{\delta}=0.73$ (ns)

$\mathrm{q}=0.73(\mathrm{~ns})$

Leisure-time:

$\beta$ at 16 years $=0.17(+)$

$\beta$ at 18 years $=0.16(+)$

Odds Ratios (5 vs. 1):

No LTPA $=1.82(+)$

Frequent LTPA $=0.59(+)$

No LTPA $=1.62$ (ns)

Frequent LTPA $=0.68$ (+ in 9 only)

Prevalence Ratios (4 vs. 1):

$\widehat{\delta}=1.29\{1.07 ; 1.56\}$ (+, ns: 3 vs. 1)

$q=1.17\{1.03 ; 1.32\}(+, n s: 2$ vs. 1$)$

$\hat{O}=1.28(1.05 ; 1.55)(+$, ns: 3 vs. 1$)$

$q=1.15(1.01 ; 1.31)(+$, ns: 2 vs. 1$)$

Manual vs. Non-manual:

$\hat{o}=1.04(0.91 ; 1.18)(\mathrm{ns})$

$q=1.14(1.05 ; 1.24)(+)$

$\widehat{\delta}=1.03\{0.90 ; 1.17\}$ (ns)
None

Age, sex, religion, marriage, urbanisation

None

Skeletal maturity, sum of skinfolds

As above plus own occupational class

None

Age

Age, own occupational class

Age

Age, own occupational class 
Table 2 Results of studies testing the association between parental occupational class and leisure-time physical activity (LTPA) in adults (Continued)

-Bowen (2010) [56]

Prevalence of vigorous exercise in manual (M)

-US; Health \& Retirement Study, and non-manual (NM) parental occupations.

$\mathrm{NM}-\mathrm{M}=6 \%(+, p<0.001)$

Study of Asset \& Health Dynamics,

\& two other cohorts

$-18,465 ; 51+$ Yrs

${ }^{a}$ Both men and women included in analysis unless otherwise stated, $N^{\hat{\gamma}}$ analytic sample consists of men only, $N^{\circ}$ analytic sample consists of women only

bLTPA leisure-time physical activity, MET metabolic equivalent, RGSC Registrar General's Social Classification (I: professional, II: managerial and technical, IIIN: skilled non-manual, IIIM: skilled manual, IV: partly skilled, V: unskilled), $M$ manual, NM non-manual

'For brevity, prevalence of LTPA shown as crude difference between named childhood SEP groups, along with measure of precision (95\% confidence intervals where available unless stated otherwise), SE standard errors, $r$ correlation coefficient, $O R$ odds ratio from logistic regression, $\beta$ : regression coefficient, " + " Statistically significant $(p \leq 0.05)$ association between less advantaged childhood SEP and less frequent adult LTP "-" Statistically significant $(p \leq 0.05)$ association between less advantaged childhood SEP and more frequent adult LTPA, $n$ s Statistically non-significant association ( $p>0.05)$ between childhood SEP and adult LTPA ${ }^{\mathrm{d}} B M I$ body mass index, CVD cardiovascular disease, $C H D$ coronary heart disease 
Table 3 Results of studies testing the association between parental education and leisure-time physical activity (LTPA) in adults

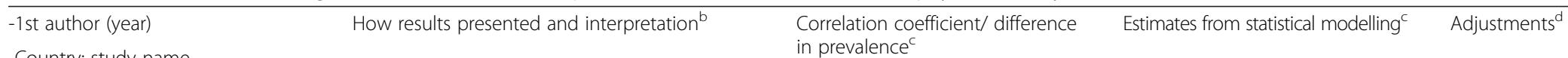

-Country; study name

in prevalence

\section{None}

-Johnson size; age

-UK; Lothian Birth Cohort 1936

Correlation and regression coefficients for

6-point LTPA score and years of parental

$r=0.08(+)$

$-1091 ; 70+$ Yrs.

-Silverwood (2012) [33]

Prevalence of LTPA (low/gardening/sport \&

-> 3100: 31-53 years

-Kuh \& Cooper (1992) [34]

-UK; MRC NSHD

-> 2850; 36 years.

$-2144 ; 36$ years.

-Pinto Pereira (2014) [35]

-UK; National Child Development Study 1958 (NCDS)

$-12,776$ had $\geq$ one measure of

LTPA; 33, 42,50 year. education. leisure), walking and cycling during work \& for pleasure (high, low) in four groups of

paternal education (1. secondary and greater 2. secondary only or primary and further education or greater 3. primary and further education with no qualifications attained 4. primary only).

$\beta=0.03$ (ns)

1-4:

Sport \& leisure $\left({ }^{\top}\right)=14.5 \%$

$(+, p<0.001)$

Sport \& leisure $(P)=20.9 \%$

$(+, p<0.001)$

Walking (High) $\left({ }^{\top}\right)=-21.6 \%$

$(-, p<0.001)$

Walking (High) $($ o $)=-8.8 \%$

$(-, p<0.001)$

Prevalence of most active in sports \& recreational activities in 4 groups of parental education

(1. secondary \& greater 2. secondary only or

primary \& further education or greater 3 . primary

$\&$ further education with no qualifications

attained 4. primary only).

Odds of most active in sport \& recreational activities comparing three highest groups of maternal education to the lowest group.

Odds of Iow LTPA comparing those with two minimally schooled parents to those without.

$1-4$

of (father) $=12 \%(+, p<0.01)$

q (father) $=21.3 \%(+, p<0.001)$

$\delta$ (mother) $=2 \%(+, p<0.001)$

o (mother) $=19 \%(+, p<0.001)$

Odds Ratios:

1 vs. $4=1.24(0.99 ; 1.55\}$ (ns)

2 vs. $4=1.52(1.22 ; 1.91\}(+)$

3 vs. $4=1.24(1.02 ; 1.50\}(+)$

Odds Ratios:

age $33=1.26\{1.15 ; 1.37\}(+)$

Age $42=1.28\{1.18 ; 1.38\}(+)$

Age $50=1.42\{1.29 ; 1.57\}(+)$

Age $33=1.14\{1.04 ; 1.26\}(+)$

Age $42=1.13\{1.03 ; 1.24\}(+)$

Age $50=1.22\{1.10 ; 1.35\}(+)$

Age $33=1.05\{0.95 ; 1.16\}$ (ns)

Age $42=1.03\{0.94 ; 1.13\}$ (ns)

Age $50=1.13\{1.01 ; 1.25\}(+)$
Own education, own

occupational class \& more

None

None

Own education, sex

childhood health, personality,

and ability at games

None

Sex

Sex, parental education,

aptitude household amenities

cognition, lifestyle factors

age $16, \&$ more 
Table 3 Results of studies testing the association between parental education and leisure-time physical activity (LTPA) in adults (Continued)

-Makinen (2009) [44]

-Finland; Health 2000 Survey $-6492 ; 30+$ Yrs.

\section{-Leino (1999) [46]}

-Finland; Cardiovascular Risk in Young Finns Study

-432; 21-30 year.

-Osler (2001) [47]

-Denmark; offspring of Copenhagen City Heart Study (CCHS).

-317; 19-31 year.
Regression coefficients for LTPA per increase in parental education (college/university/ $>12$ years, high/comprehensive school/ 12 years, high school/10 year, 1 year of technical college/8-9 years, elementary school/7 years).

Odds of inactivity and moderate LTPA relative to high LTPA by parental education (secondary, middle, primary) with secondary education used as reference category.

Prevalence of physical inactivity in three groups of parental education (1.>12 years 2. 9-12 years 3.<9 years).

$1-3\left(\delta^{\top}\right)=-14.7 \%(\mathrm{~ns})$

$$
1-3(\text { (q) }=-9.2 \% \text { (ns) }
$$

Age $33=1.02\{0.92 ; 1.13\}$ (ns)

Age $42=1.00\{0.91 ; 1.10\}$ (ns)

Age $50=1.07\{0.96 ; 1.19\}$ (ns)

$\beta$ (estimated from figures):

Age 25 (father) $\approx 0.06$ (ns)

Age 33 (father) $\approx 0.12(+)$

Age 40 (father) $\approx 0.01$ (ns)

Age 25 (mother) $\approx 0.05$ (ns)

Age 33 (mother) $\approx 0.12(+)$

Age 40 (mother) $\approx-0.06$ (ns)

Age 25 (father) $\approx 0.01$ (ns)

Age 33 (father) $\approx 0.05$ (ns)

Age 40 (father) $\approx 0.01$ (ns)

Age 25 (mother) $\approx-0.01$ (ns)

Age 33 (mother) $\approx 0.06$ (ns)

Age 40 (mother) $\approx-0.01$ (ns)

ORs (primary vs. secondary):

Inactivity $\left({ }^{\widehat{O}}\right)=1.10(\mathrm{~ns})$

Inactivity $($ ( ) $)=1.56(+)$

Moderate LTPA $($ ふ $)=1.45(\mathrm{~ns})$

Moderate LTPA $(\%)=1.37$ (ns)

Age

$\begin{array}{ll}\text { Odds Ratios (1 vs. 3): } & \text { None } \\ \hat{O}=1.3\{0.6 ; 3.0\} \text { (ns) } & \\ \hat{q}=0.5\{0.2 ; 1.1\} \text { (ns) } & \\ \hat{\delta}=0.7\{0.4 ; 3.2\} \text { (ns) } & \begin{array}{l}\text { Age, own education, own } \\ \text { occupational class, } \\ \text { smoking status }\end{array}\end{array}$

As above plus own education, own social class, BMl, mental health, number of children in the

Sex, whether participated in school health education intervention

As above plus own education 
Table 3 Results of studies testing the association between parental education and leisure-time physical activity (LTPA) in adults (Continued)

\begin{tabular}{|c|c|c|c|c|}
\hline -Beunen (2004) [50] & \multirow{4}{*}{$\begin{array}{l}\text { Correlation between sports, leisure-time and } \\
\text { counts indices of physical activity and } \\
\text { parental education. }\end{array}$} & \multicolumn{2}{|l|}{$r$ (sport, father) $=0.17(+)$} & \multirow[t]{4}{*}{ None } \\
\hline -Belgium; LLSFB & & $r$ (sport, mother) $=0.14(\mathrm{~ns})$ & & \\
\hline \multirow[t]{4}{*}{$-166^{\hat{A}} ; 40$ year. } & & $r$ (leisure-time, father) $=0.14$ (ns) & & \\
\hline & & $r$ (counts, mother) $=0.15(\mathrm{~ns})$ & & \\
\hline & \multirow{2}{*}{$\begin{array}{l}\text { Regression coefficients for sport, leisure-time } \\
\text { and counts indices of physical activity per } \\
\text { increase in years of parental education }\end{array}$} & & $\beta$ (sport, father) $=0.19(+)$ & Stature (sport index) \\
\hline & & & $\beta$ (leisure-time, father) $=0.14(+)$ & $\begin{array}{l}\text { Stature, pulse recovery } \\
\text { (leisure-time index) }\end{array}$ \\
\hline -Wray (2005) [55] & \multirow{5}{*}{$\begin{array}{l}\text { Odds of low physical activity per unit increase } \\
(0-17) \text { in years of parental education. }\end{array}$} & & Odds Ratios: & \\
\hline \multirow{2}{*}{$\begin{array}{l}\text {-US; Health \& Retirement Study } \\
\text { (HRS); Study of Asset \& Health } \\
\text { Dynamics (AHEAD) }\end{array}$} & & & $\mathrm{HRS}=0.964(+, p \leq 0.001)$ & \multirow{2}{*}{$\begin{array}{l}\text { Age, gender, ethnicity, } \\
\text { marriage, interactions }\end{array}$} \\
\hline & & & AHEAD $=0.878(+, p \leq 0.001)$ & \\
\hline \multirow{2}{*}{$\begin{array}{l}-6106 ; 51-61 \text { year (HRS), 3636; } \\
\text { 70+ Yrs. (AHEAD) }\end{array}$} & & & $\mathrm{HRS}=0.976(+, p \leq 0.05)$ & \multirow{2}{*}{$\begin{array}{l}\text { As above plus own } \\
\text { education, economic } \\
\text { resources }\end{array}$} \\
\hline & & & AHEAD $=0.910(+, p \leq 0.05)$ & \\
\hline -Bowen (2010) [56] & \multirow{3}{*}{$\begin{array}{l}\text { Prevalence of vigorous exercise in two groups } \\
\text { of parental education ( } 1 .>8 \text { years } 2 . \leq 8 \text { years). }\end{array}$} & $1-2$ (father) $=4 \%(+, p \leq 0.001)$ & & \multirow[t]{3}{*}{ None } \\
\hline -US; HRS,AHEAD \& more & & $1-2$ (mother) $=4 \%(+, p \leq 0.001)$ & & \\
\hline$-18,465 ; 51+$ Yrs. & & & & \\
\hline -Phillips (2009) [61] & \multirow{3}{*}{$\begin{array}{l}\text { Correlation between exercise kilocalories/week. } \\
\text { and years (1-24) of parental education. }\end{array}$} & \multirow[t]{3}{*}{$r=0.084(+)$} & & \multirow[t]{3}{*}{ None } \\
\hline -US; Health \& Behaviour Project & & & & \\
\hline$-811 ; 30-54$ years. & & & & \\
\hline -Frank (2003) [58] & \multirow{3}{*}{$\begin{array}{l}\text { Prevalence of exercise in six groups of parental } \\
\text { education (1. medical school } 2 \text {. graduate } \\
\text { school 3. college graduate } 4 \text {. some college } \\
\text { 5. high school } 6 \text {. < High school) and three } \\
\text { groups of both parent's education) (1. Both } \geq \\
\text { graduate school } 2 \text {. mix 3. Both } \leq \text { graduate } \\
\text { school). }\end{array}$} & $1-6$ (father) = $2 \%$ (ns) & & \multirow[t]{3}{*}{ None } \\
\hline -US; Women Physician Health Study & & $1-6$ (mother) $=-4 \%$ (ns) & & \\
\hline$-2884^{\circ} ; 30-70$ year. & & $1-3$ (both) $=5 \%$ (ns) & & \\
\hline -Gall (2010) [64] & \multirow{3}{*}{$\begin{array}{l}\text { Prevalence of LTPA by level of parental } \\
\text { education (1. high 2. medium 3. low). }\end{array}$} & $1-3:$ & & \multirow[t]{3}{*}{ None } \\
\hline \multirow{2}{*}{$\begin{array}{l}\text {-Australia; Childhood Determinants } \\
\text { of Adult Health Study }\end{array}$} & & \multirow{2}{*}{$\begin{array}{l}\hat{\sigma}=3 \% \text { (ns) } \\
q=1 \% \text { (ns) }\end{array}$} & & \\
\hline & & & & \\
\hline
\end{tabular}

Both men and women included in analysis unless otherwise stated, $N^{\hat{\beta}}$ analytic sample consists of men only, $N^{+}$analytic sample consists of women only

b LTPA leisure-time physical activity

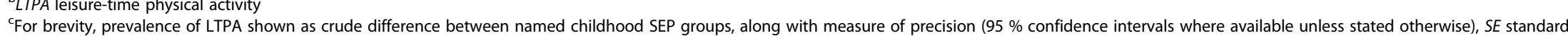
errors, $r$ correlation coefficient, OR odds ratio from logistic regression, $\beta$ regression coefficient, "+" Statistically significant $(p \leq 0.05)$ association between less advantaged childhood SEP and less frequent adult LTPA, "_" Statistically significant $(p \leq 0.05)$ association between less advantaged childhood SEP and more frequent adult LTPA, $n$ s Statistically non-significant association $(p>0.05)$ between childhood SEP and adult LTPA $\mathrm{d}_{B M I}$ body mass index 
Table 4 Results of studies testing the association between indices and other measures of childhood socioeconomic position and leisure-time physical activity (LTPA) in adults -1st author (year) How results presented and interpretation ${ }^{\mathrm{b}}$ Correlations coefficient/difference Estimates from statistical modelling ${ }^{c}$ Adjustments

-Country; study name in prevalence ${ }^{c}$

\section{mple size; age}

-Johnson (2011) [20]

Correlation and regression coefficients for a

6-point LTPA score and an index of

-UK; Lothian Birth Cohort 1936

-1091; 70 year.

-Hilsdon (2008) [22]

-UK; British Women's Heart

$\&$ Health Study (BWHHS)

-> 4100 ; 60-79 years.

-Watt (2009) [23]

$-U K$; BWHHS

$-3523^{\circ} ; 60-79$ years.

-Pinto Pereira (2014) [35]

-UK; National Child

Development Study

1958 (NCDS)

$-12,776$ had $\geq$ one measure

of LTPA; 33, 42, 50 year.
Prevalence of four indicators of childhood household amenities and car access in

4 groups of frequency of physical activity

hours/week.

Odds of more frequent physical activity per unit increase in childhood SEP (parental occupation, household amenities and car access) with higher scores representing more adversity.

Difference in prevalence of low exercise between those reporting no and those reporting yes to questions on childhood household amenities and car access.

Odds of low exercise per unit increase in childhood SEP with higher scores representing more adversity.

Odds of low LTPA per unit increase (0-18) on index of childhood household amenities (access to bathroom, indoor lavatory and hot water, with higher scores indicating more limited access).

$r=0.00$ (ns)

23-0 (hours/week.):

Shared bedroom $=-7.7 \%\{-5.9 ;-8.7\}(+)$

No indoor toilet $=-8.8 \%\{-7.9 ;-9.8\}(+)$

No hot water $=-9.6 \%\{-8.6 ;-10.4\}(+)$

No car access $=-7.9 \%\{-6.8 ;-9.1\}(+)$

$\mathrm{OR}=0.85\{0.81 ; 0.89\}(+)$
$\mathrm{OR}=0.93\{0.89 ; 0.98\}(+)$
$\mathrm{OR}=0.94\{0.90 ; 0.99\}(+)$

Shared bedroom $=5.4 \%\{1.9 ; 9.0\}(+)$

No hot water $=6.1 \%\{2.4 ; 9.8\}(+)$

No indoor toilet $=6.8 \%\{3.1 ; 10.4\}(+)$

No car access $=7.9 \%\{3.3 ; 12.4\}(+)$

$\mathrm{OR}=1.12\{1.07 ; 1.17\}(+)$

$\mathrm{OR}=1.06\{1.01 ; 1.12\}(+)$

Odds ratios:

Age $33=1.03\{1.01 ; 1.04\}(+)$

Age $42=1.03\{1.01 ; 1.04\}(+)$

Age $50=1.04\{1.03 ; 1.05\}(+)$

Age $33=1.02\{1.001 ; 1.03\}(+)$

Age $42=1.01\{0.999 ; 1.03\}$ (ns)

Age $50=1.02\{1.01 ; 1.04\}(+)$

None

Own education, own

occupational class \& more

None

Age

Age, adult SEP, area deprivation.

As above plus smoking $\mathrm{BMI}, \mathrm{CVD}$, respiratory disease

None

None

Age, own adult SEP

None 
Table 4 Results of studies testing the association between indices and other measures of childhood socioeconomic position and leisure-time physical activity (LTPA) in adults (Continued)

$$
\begin{aligned}
& \text { Age } 33=1.01\{0.995 ; 1.03\} \text { (ns) } \\
& \text { Age } 42=1.01\{0.99 ; 1.02\} \text { (ns) } \\
& \text { Age } 50=1.02\{1.002 ; 1.03\} \text { (+) } \\
& \text { Age } 33=1.01\{0.99 ; 1.02\} \text { (ns) } \\
& \text { Age } 42=1.01\{0.99 ; 1.02\} \text { (ns) } \\
& \text { Age } 50=1.01\{0.999 ; 1.03\} \text { (ns) }
\end{aligned}
$$

No conditioning activity: Prevalence of conditioning inactivity \&

-Lynch (1997) [39]

-Finland; Kuopio Ischaemic Heart Disease Risk Factor Stud

$-2682^{\delta} ; 42-60$ year.

-Makinen (2009) [44]

-Finland; Health 2000 Survey

$-6492 ; 30+$ Yrs.

-Beunen (2004) [50]

-Belgium; LLSFB

$-166^{\hat{\imath}} ; 40$ year

-Scheerder (2006) [51]

-Belgium; Leuven Longitudinal Study of Flemish Girls (LLSFG)

$-234^{\circ} ; 32-41$ year.

-Pudrovska (2013) [54]

-US; 1957 Wisconsin

Longitudinal Study

-5778 ; 65 years.
Prevartile of conditioning activity by

an index of parental occupation, parenta

education \& more (1. high 2. middle

3. poor).

Odds of inactivity and moderate LTPA relative to high LTPA for those reporting yes to long-term financial problems; regular parental unemployment.

Correlation and regression coefficients for sport, leisure-time and counts indices per increase in urbanisation score of the childhood home. Only counts results presented in paper.

Path coefficients for level of sports participation based on an index of parental occupation and parental parentaloccupation and parental , middle class, upper class).

Path coefficients for exercise per change in index of parental occupation, parental education, family income, father's

occupational income and occupational education
$1-3=-0.4 \%$ (ns)

Low quartile:

$$
1-3^{+}=-5.7 \%(+)
$$

Odds Ratios (inactivity):

$\delta=1.04$ (ns); 1.35 (ns)

q $=1.18$ (ns); 1.45 (ns)

Odds Ratios (moderate LTPA):

$\widehat{\delta}=0.95$ (ns); 1.31 (ns)

q $=1.13$ (ns); 1.36 (ns)

Counts:

$r=0.18(+)$

Counts:

$\beta$ at 14 years $=0.17(+)$

$\beta$ at 16 years $=0.15(+)$

$\beta$ at 18 years $=0.15(+)$

$\beta$ from path model $=-0.07\{-0.22 ; 0.08\}$ (ns) Age, own education, own

'Total effects'

$\beta=1.117(+, p<0.001)$

'Direct effects'

$\widehat{\delta}=0.211(+, p<0.01)$

$q=0.091(+, p<0.05)$

$\widehat{o}=0.018$ (ns)

q $=0.039$ (ns) occupational class, BM! parent's sport, \& more

None

Sex, parental education,

household amenities,

cognition, aptitude, lifestyle

fors at age 16, \& more

As above plus own education, own social class, BMl, mental health, number of children in the household, limiting ilness

Age

Age

Sit reach, pulse recovery, sports participation

(regression)

Marriage, children, alcoho use, smoking status, own SES, health, obesity,

depression

As above plus high school sports 
Table 4 Results of studies testing the association between indices and other measures of childhood socioeconomic position and leisure-time physical activity (LTPA) in adults (Continued)

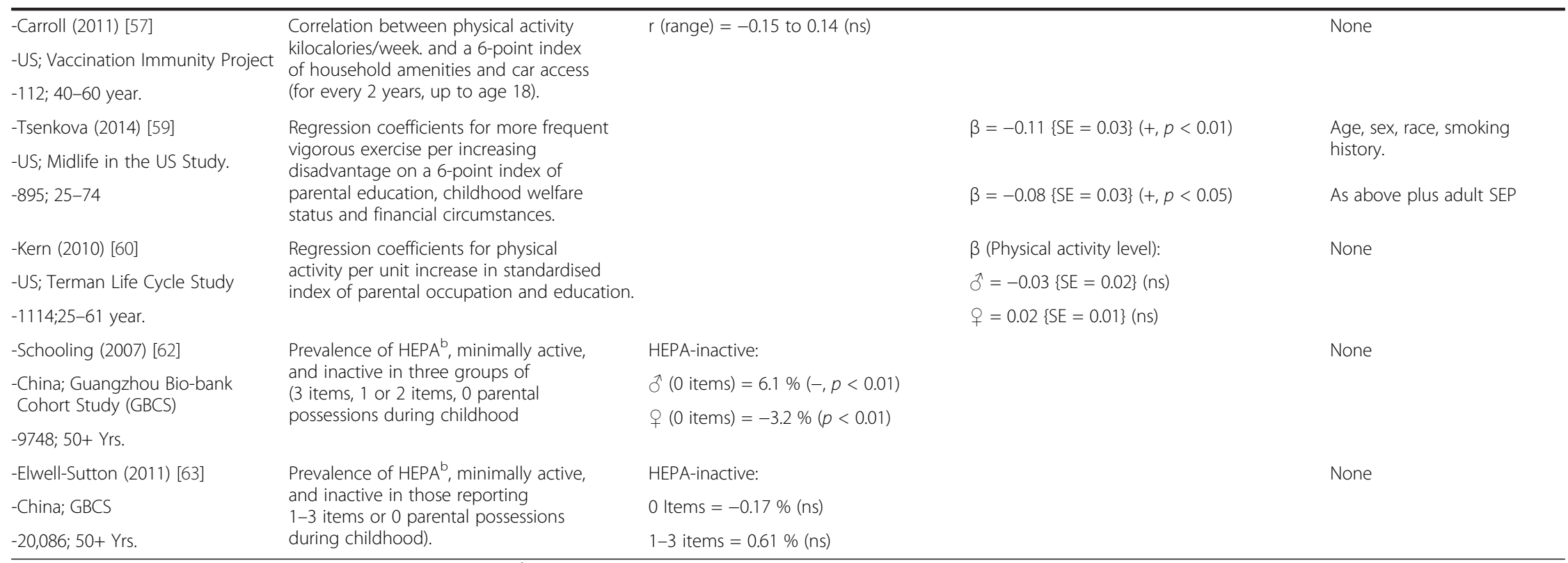

\section{${ }^{a}$ Both men and women included in analysis unless otherwise stated, $N^{3}$ analytic sample consists of men only, $N^{+}$analytic sample consists of women only}

bLTPA leisure-time physical activity, HEPA Health enhancing physical activity-acronym used in the two GBCS papers $[62,63]$

${ }^{C}$ For brevity, prevalence of LTPA shown as crude difference between named childhood SEP groups, along with measure of precision (95\% confidence intervals where available unless stated otherwise), SE standard errors, $r$ correlation coefficient, OR odds ratio from logistic regression, $\beta$ regression coefficient; " + " Statistically significant $(p \leq 0.05)$ association between less advantaged childhood SEP and less frequent adult LTPA, "- - "Statistically significant $(p \leq 0.05)$ association between less advantaged childhood SEP and more frequent adult LTPA, $n s$ Statistically non-significant association $(p>0.05)$ between childhood SEP and adult LTPA ${ }^{\mathrm{d}} B M I$ body mass index, CVD cardiovascular disease 
Manual father's occupation was associated with less LTPA and more inactivity in men [24, 25] and women [21-23] from the British Regional Heart Study (BRHS) and British Women's Heart and Health Study (BWHHS) respectively. After accounting for age and own occupational class [21, 25], this association was only found in the BWHHS [21]. A higher prevalence [28] and higher odds [26] of inactivity were reported in lower parental occupational groups of the Whitehall II Study. This association was considerably attenuated and no longer significant following adjustment for adult SEP [28] and a third paper from this cohort reported no difference in levels of inactivity between manual and non-manual parental occupations [27]. Findings from the West of Scotland Collaborative Study suggest less exercise in lower parental occupations $[29,30]$. A weak correlation between higher paternal occupational groups and more LTPA was reported in the Lothian Birth cohort 1936 (LBC1936) and no association was found in analysis adjusted for adult SEP [20]. In the Mid-span family study, manual and non-manual groups did not differ by levels of inactivity [32], but the prevalence of sports and exercise was higher in higher father's occupational groups of a Scottish survey [31].

Several Scandinavian studies reported null findings including Danish [38] and Finnish [43] birth cohorts, a Norwegian study [45], and an analysis of 34 year old Swedes [42]. The latter [42] found that women but not men from non-manual paternal backgrounds spent more metabolic equivalent hours/week in LTPA compared with those of manual father's occupations. Higher and lower father's occupational groups were less and more respectively, inactive than the mean level of activity of employed Swedes [48], but this was not tested at a high significance level $(p<0.10)$ [48]. Mostly null findings were reported in the Finnish Health 2000 Survey [44] however, men from lower paternal occupational groups were found to be more inactive than those from higher groups and women with mothers in manual occupations were more likely to be only moderately active compared with daughters of office employee mothers [44].

Dutch adults living near Eindhoven from lower paternal occupational strata were more likely to be inactive and less likely to be frequently active during leisure-time compared with those from professional backgrounds [53]. After accounting for own occupational class, this association remained for frequent LTPA and in women only [53]. A Dutch study that only included men from Eindhoven [52] found no difference by parental occupation in the prevalence of activity [52]. Belgian men's paternal occupational class was associated with their leisure-time but not sports or accelerometer indices [50]. Age-adjusted findings from an older Spanish sample showed that lower father's occupational groups were more likely to be inactive than higher groups and the association was more evident in women following adjustment for own occupational class [49]. Compared with the manual group, non-manual father's occupational groups of a large US sample had a higher prevalence of vigorous exercise [56].

\section{Parental education}

Fourteen papers (13 studies) presented associations for parental years or level of education and ten (9 studies) found evidence of an association between lower levels of parental education and less frequent LTPA in adults (Table 3).

Similar trends to those found for occupation were reported in the NSHD, i.e. less LTPA [33, 34] and more walking (during work and pleasure) [33] in lower parental educational groups. Analysis adjusted for own education showed that those with more highly educated mothers were more active in sports at age 36 [34] but no difference was found when the highest maternal educational group was compared to the lowest (Table 3). Gender-adjusted NCDS analyses comparing those without and with two minimally educated parents showed that the latter were more likely to be physically inactive at ages 33,42 and 50 years [35]. This association was fully attenuated at ages 33 and 42 after other early life factors were included in the analysis and likewise at age 50 following further adjustments including for own adult SEP (Table 3). More parental years in education were weakly correlated with more LTPA in LBC1936 but adjusted analysis did not find an association [20].

Parental education was unrelated to leisure-time inactivity in a Danish sample [47], and null-findings were reported by two Finnish studies [44, 46]. However, one of the latter [44] found that Finnish women with a primarylevel educated parent were more likely to be inactive compared with those with a secondary-level educated parent [44]. LTPA (at age 33 only) was linked to parental education in a Norwegian study but not after adjustment for own education [40]. Belgian men's father's education was related to their self-reported sports and leisure-time activity but not accelerometer indices [50]. A lower parental education in US adults was associated with less prevalent vigorous exercise [56] and with higher adjusted-odds of low exercise [55]. Higher parental education was correlated with higher estimated exercise energy expenditure in a Pennsylvanian sample [61]. Three measures of parental education were unrelated to exercise in women physicians born in the US [58] and there was no association between parental education and LTPA in an Australian study [64].

\section{Indices and other measures of childhood SEP}

Fourteen papers (12 studies) tested associations between indices and other measures of childhood SEP and adult LTPA and seven (6 studies) found an association between a lower childhood SEP and less frequent LTPA in adulthood (Table 4). 
An index measuring household characteristics and car access during childhood was unrelated to LTPA in LBC1936 [20] but four measures of housing characteristics and car access were each associated with LTPA in the BWHHS $[22,23]$. Combining these four indicators and paternal occupation into a summary variable showed that with increasing childhood socioeconomic adversity, women were more likely to be low exercisers [23] and less likely to be more physically active [22]. Having more limited household amenities was related to leisure-time physical inactivity at ages 33, 42 and 50 years in NCDS, but not at age 42 when gender was taken into account [35]. After adjustment for a range of early life factors, this association was only found at age 50 and associations were considerably attenuated and were no longer observed at any age following the addition of adult covariates, including own SEP, to the analysis (Table 4).

Compared with those ranked middle or poor on an index of parental occupation, education, external perceptions of wealth, and housing characteristics, Finnish men who ranked high on the index were less likely to be in the lowest quartile of conditioning activities [39]. No difference was found when the prevalence of inactivity was compared in this sample [39]. A different Finnish study reports no association between long-term financial problems or regular parental unemployment and LTPA in adults [44]. More urban locations of Belgian men's childhood homes were related to higher accelerometer counts but not to any self-reported outcomes [50]. An index of parental occupation and education was not associated with Belgian women's sports participation [51].

Increasing disadvantage as indicated by an index of parental education, childhood welfare status and financial level growing up was associated with less participation in vigorous exercise in US adults [59]. This association was only partially attenuated following adjustment for own adult SEP [59]. An index of parental occupation and education was unrelated to activities and hobbies of a Californian sample [60], but in older US adults [54] a higher childhood SEP, indicated by a similar index that included parental income, was associated with more exercise at age 65 . The authors tested the role of mediating factors and report that own SEP explained almost half of this association [54]. No correlation was found in 112 US participants between a similar index and estimated activity energy expenditure [57]. Findings from the GBCS suggest a higher prevalence of inactivity (and a lower prevalence of LTPA) in Chinese participants reporting more parental possessions during their childhood $[62,63]$.

\section{Discussion}

\section{Summary of results}

This systematic review included 45 papers from 36 study samples and found evidence of less frequent LTPA in adults from less advantaged childhood socioeconomic backgrounds. Twenty-two studies report results that associate a lower childhood SEP with less frequent adult LTPA; thirteen studies report no association. 9/16 studies that presented results adjusted for own adult SEP reported statistically significant associations between childhood SEP and adult LTPA [21-23, 34, 35, 42, 49, 53-55, 59]. Studies presenting results before and after adjustment for adult SEP found that accounting for own SEP in adulthood typically partly attenuated associations (Tables 2, 3, 4). Gender-stratified analyses showed more evidence of an association in women compared with men [34, 42, 44, 49, 53]. $1 / 10$ UK [32], 3/8 US [57, 58, 60] and 6/11 Scandinavian $[38,43,45-48]$ studies found no evidence of childhood socioeconomic differences in adults' LTPA. Findings did not differ systematically by type of childhood SEP indicator or age at assessment of LTPA.

\section{Explanation of findings}

Existing reviews link a lower childhood SEP to a range of disadvantageous adult outcomes, including physical capability [65], cardiovascular disease [66] and mortality [67]. Reviews focusing on different life stages have shown that from childhood through to old age, in crosssectional analyses, lower socioeconomic groups tend to participate less in LTPA than more advantaged groups [7-9]. In addition to participating less in LTPA during childhood [7], a study of over 2000 Dutch adults provides evidence that children from lower socioeconomic backgrounds have a lower likelihood of initiating a sport throughout their lives [68].

One possible reason for finding an association between a lower childhood SEP and less frequent adult LTPA is due to the continuity of SEP across life. A lower childhood SEP tends to restrict future SEP [69], partly by predisposing to social pathways operating across life which can limit educational opportunities and ultimately socioeconomic potential, e.g. in occupational class, income and wealth [10]. These pathways can influence the availability of, and a person's response to, opportunities for the development of LTPA [10].

Associations between childhood SEP and adult LTPA were reported in several analyses which were adjusted for own adult SEP $[21-23,34,35,42,49,53-55,59]$ suggesting that complementary pathways are likely to be involved (Fig. 2). Participation in sports and exercise in early life tends to be socioeconomically patterned [7] and tracks into adulthood [70], potentially forming an important determinant of adult LTPA. Since adult LTPA also displays a socioeconomic gradient $[8,9]$, less socioeconomically advantaged children are likely to have less physically active parents who may in turn unfavourably influence their own children's involvement in LTPA [71]. Childhood socioeconomic circumstances may influence the acquisition 


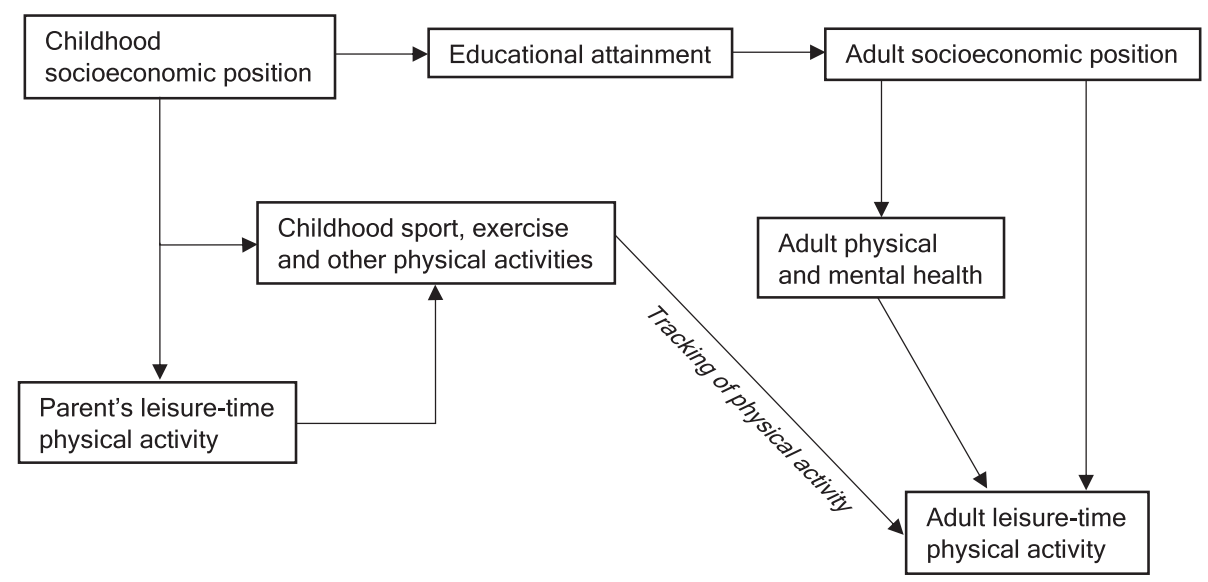

Fig. 2 Hypothesised pathways explaining associations found between childhood socioeconomic position and adult leisure-time physical activity

of sets of interpersonal skills such as decision making, self-efficacy and self-esteem which can help people maintain health behaviour such as LTPA [72]. Socioeconomic differences in children's growth and motor development [73] could contribute to differences in subsequent LTPA.

Thirteen studies presenting only null findings do not support the review's hypothesis [32, 38, 43, 45-48, 51, $52,57,58,60,64]$. Participation in sports and exercise is linked to a range of factors other than SEP, including genetics [74], life transitions, culture and policy [75], some of which could play a greater role in determining participation. Evidence for less tracking of LTPA when compared with other health behaviours such as sedentary behaviour [76] supports this argument although measurement error could explain the lower tracking of LTPA [70]. Associations may vary by setting and cohort due to varying influences on LTPA by these factors and are also likely to be influenced by study quality.

\section{Sources of heterogeneity}

Inconsistent findings could be due to differences between studies including in design and risk of bias. Despite an overall medium study quality, considerable variation between studies in the assessment and formulation of LTPA (Table 1) and adjustment for potential confounders (Tables 2, 3, 4) can influence associations. Small sample sizes $[47,50,51]$ may lead to underpowered studies while multiple tests $[40,44,50]$ risk detecting false associations. Lack of an association in men from the BRHS after accounting for adult SEP [25], and other reported null findings [30, 32], may be due to heterogeneity within childhood SEP groups as a result of using dichotomous indicators. Null findings from the Women Physician Health Study [58] might reflect insufficient variation in childhood socioeconomic background.
We did not find that results varied by the method of ascertainment of childhood SEP however, using recalled measures of childhood SEP can underestimate associations [77]. There was little evidence that the type of childhood SEP indicator used was a source of heterogeneity, suggesting that each indicator sufficiently captures the same underlying construct or that the various aspects of SEP are equally important. This is a similar observation to that of a previous review of European adults [9] but contrary to an earlier and geographically wider review which found education to be more strongly associated with contemporaneous LTPA [8].

Genuine gender differences in the association between childhood SEP and adult LTPA might exist. Like some studies in this review, a previous review found more evidence of an association in women than men between adult SEP and LTPA [8]. Absence of a gender difference in how childhood SEP relates to adult's capacity to undertake exercise [65] suggests that the gender differences found in this review are likely explained by social rather than biological pathways, such as differences in risk factors which could impact on subsequent LTPA [78].

The tendency for Scandinavian studies to find less evidence of association compared with UK studies might be due to less variation between socioeconomic groups in Scandinavian cohorts than in the UK [79]. There could also be differences in the meaning of occupation between these settings, e.g. in Scandinavian cohorts, where there was more prevalent farming occupations [43-45, 48], SEP could be indicating how urban or rural is the environment, which may be independently related to LTPA [80].

More walking during work and pleasure in lower childhood SEP groups of the NSHD [33] might be explained by the inclusion of work-related PA as part of the outcome, which can be inversely associated with SEP [9]. Socioeconomic patterns of LTPA that are different to those usually 
observed in Western countries have been documented in China [81], which could explain the GBCS findings [62]. Other cohort, period and cultural differences might explain some of the between-study heterogeneity.

\section{Implications of findings}

Due to heterogeneity in findings, a better understanding of how childhood SEP relates to adult LTPA is required. Future studies should use prospectively ascertained indicators of childhood SEP where this is feasible and examine validated and reliable measures of LTPA. Data from PA monitors could be used in conjunction with questionnaires to derive more holistic LTPA variables that capture parameters such as activity type, energy expenditure and time of day/week that activity is performed [82]. Strategies for maximising participant retention in long-running studies should be considered so as to minimise bias due to loss to follow-up. Within-individual levels of LTPA can vary over time and future research could in addition explore associations with patterns or change in LTPA, as well as different types of LTPA. To better characterise how associations vary by time and place, age, country, cohort and period differences should be formally tested while accounting for methodological differences. Testing hypothesised pathways (Fig. 2) can aid our knowledge of how childhood SEP relates to adult LTPA.

Despite the inconsistencies described, childhood socioeconomic circumstances can influence health throughout life and interventions to improve them will likely lead to additional benefits besides increased adult LTPA. As well as improving early life circumstances, intervening to promote adult LTPA could be one means to cut the link between a disadvantaged childhood SEP and poor adult health. Effectively promoting adult LTPA amongst those disadvantaged in childhood may in turn require a better understanding of the mechanisms linking childhood disadvantage to adult LTPA.

\section{Strengths and limitations of the review}

Strengths of this review are the systematic process followed to locate and extract data from eligible studies and the searching of multiple databases and reference lists. Independently working researchers helped reduce the potential for errors in study screening, data extraction and quality assessment. Limitations include search restrictions to English language and to journal publications, which may introduce publication bias. The fact that presented results were not sufficiently comparable to be combined in a meta-analysis could be considered a limitation and this also meant we could not formally assess publication bias. However, the inclusion of all studies even where the review's question was not the primary aim, and the findings of no association between childhood SEP and adult LTPA in 13 studies suggests publication bias is unlikely.

\section{Conclusions}

This systematic review found evidence of an association between a less advantaged SEP in childhood and less frequent LTPA in adults (particularly among women and in UK cohorts) but considerable heterogeneity between studies was detected. Future studies should examine more detailed measures of LTPA, investigate underlying pathways and explore country differences. The findings suggest the need to provide additional opportunities and support to enable children from socioeconomically disadvantaged backgrounds to develop and maintain more active leisure pursuits and participate in sports and exercise across life.

\section{Additional files}

Additional file 1: Search terms.

Additional file 2: Data extraction form.

Additional file 3: Quality assessment form.

\section{Abbreviations}

PA: Physical activity; LTPA: Leisure-time physical activity; SEP: Socioeconomic position; PRISMA: Preferred Reporting Items for Systematic Reviews and Meta-Analysis.

\section{Competing interests}

The authors declare that they have no competing interests.

\section{Authors' contributions}

$A E, R C, D B$ and $R H$ designed the review and carried out study selection, data extraction and quality assessment. AE drafted the first version of the manuscript. All authors read and approved the final version.

\section{Acknowledgments}

The study is funded by the MRC (programme code MC_UU_12019/1). The funder had no role in the design of the study or the writing of the manuscript and played no part in the decision to submit it for publication.

\section{Author details}

${ }^{1} \mathrm{MRC}$ Unit for Lifelong Health and Ageing at UCL, 33 Bedford Place, London WC1B 5JU, UK. ${ }^{2}$ Centre for Longitudinal Studies, UCL Institute of Education, 20 Bedford Way, London WC1H OAL, UK.

Received: 13 April 2015 Accepted: 17 June 2015

Published online: 03 July 2015

\section{References}

1. Warburton DE, Nicol CW, Bredin SS. Health benefits of physical activity: the evidence. CMAJ. 2006;174(6):801-9.

2. Lee IM, Shiroma EJ, Lobelo F, Puska P, Blair SN, Katzmarzyk PT. Lancet Physical Activity Series Working Group. Effect of physical inactivity on major non-communicable diseases worldwide: an analysis of burden of disease and life expectancy. Lancet. 2012;380(9838):219-29.

3. Bélanger $M$, Townsend $N$, Foster $C$. Age-related differences in physical activity profiles of English adults. Prev Med. 2011;52(3-4):247-9.

4. Andersen $\mathrm{K}$, Mariosa D, Adami HO, Held C, Ingelsson E, Lagerros YT, et al. Dose-response relationship of total and leisure time physical activity to risk of heart failure: a prospective cohort study. Circ Heart Fail. 2014;7(5):701-8.

5. Ekelund U. Lifetime lifestyles II: physical activity, the life course, and ageing. In: Kuh D, Cooper R, Hardy R, Richards M, Ben-Shlomo Y, editors. A life course approach to healthy ageing. 1st ed. New York: Oxford University Press; 2014. p. 229-45. 
6. Conn VS, Hafdahl AR, Mehr DR. Interventions to increase physical activity among healthy adults: meta-analysis of outcomes. Am J Public Health. 2011;101(4):751-8.

7. Stalsberg R, Pedersen AV. Effects of socioeconomic status on the physical activity in adolescents: a systematic review of the evidence. Scand J Med Sci Spor. 2010;20:368-83.

8. Gidlow C, Johnston LH, Crone D, Ellis N, James D. A systematic review of the relationship between socio-economic position and physical activity. Health Educ J. 2006;65(4):338-67.

9. Beenackers MA, Kamphuis CB, Giskes K, Brug J, Kunst AE, Burdorf A, et al. Socioeconomic inequalities in occupational, leisure-time, and transport related physical activity among European adults: a systematic review. Int J Behav Nutr Phys Act. 2012;9:116.

10. Kuh D, Power C, Blane D, Bartley M. Socioeconomic pathways between childhood and adult health. In: Kuh D, Ben-Shlomo Y, editors. A life course approach to chronic disease epidemiology. 2nd ed. New York: Oxford University Press; 2004. p. 371-98.

11. Tammelin T. A review of longitudinal studies on youth predictors of adulthood physical activity. Int J Adolesc Med Health. 2005;17(1):3-12.

12. Moher D, Liberati A, Tetzlaff J, Altman DG. Preferred reporting items for systematic reviews and meta-analyses: the PRISMA statement. BMJ. 2009;339:b2535.

13. Elhakeem A, Cooper R, Bann D, Hardy R. Childhood socioeconomic position and adult leisure-time physical activity: a systematic review protocol. Syst Rev. 2014;3:141.

14. Krieger N, Williams DR, Moss NE. Measuring social class in US public health research: concepts, methodologies, and guidelines. Annu Rev Public Health. 1997;18:341-78.

15. Galobardes B, Shaw M, Lawlor DA, Lynch JW, Davey SG. Indicators of socioeconomic position (part 1). J Epidemiol Community Health. 2006;60(1):7-12.

16. Howley ET. Type of activity: resistance, aerobic and leisure versus occupational physical activity. Med Sci Sports Exerc. 2001;33(6 Suppl):S364-9.

17. United Nations Department of Economic and Social Affairs. Definition of youth. http://www.un.org/esa/socdev/documents/youth/fact-sheets/youthdefinition.pdf. Accessed 08 Jun 2015.

18. The Newcastle-Ottawa Quality Assessment Scale. http://www.ohri.ca/ programs/clinical_epidemiology/nosgen.pdf. Accessed 10 Jan 2014.

19. Sanderson S, Tatt ID, Higgins JP. Tools for assessing quality and susceptibility to bias in observational studies in epidemiology: a systematic review and annotated bibliography. Int J Epidemiol. 2007;36(3):666-76.

20. Johnson W, Corley J, Starr JM, Deary IJ. Psychological and physical health at age 70 in the Lothian Birth Cohort 1936: links with early life IQ, SES, and current cognitive function and neighborhood environment. Health Psychol. 2011;30:11.

21. Lawlor DA, Davey Smith G, Ebrahim S. Association between childhood socioeconomic status and coronary heart disease risk among postmenopausal women. Findings from the British Women's, Heart and Health Study. Am J Public Health. 2004;94:1392.

22. Hillsdon M, Lawlor DA, Ebrahim S, Morris JN. Physical activity in older women: associations with area deprivation and with socioeconomic position over the life course: observations in the British Women's Heart and Health Study. J Epidemiol Community Health. 2008;62:350.

23. Watt HC, Carson C, Lawlor DA, Patel R, Ebrahim S. Influence of life course socioeconomic position on older women's health behaviors: findings from the British Women's Heart and Health Study. Am J Public Health. 2009:99:320-7.

24. Ramsay SE, Whincup PH, Morris RW, Lennon LT, Wannamethee SG. Are childhood socio-economic circumstances related to coronary heart disease risk? Findings from a population-based study of older men. Int J Epidemiol. 2007;36:566

25. Wannamethee SG, Whincup PH, Shaper G, Walker M. Influence of fathers' social class on cardiovascular disease in middle-aged men. Lancet. 1996;348(9037):1259-63.

26. Stringhini S, Batty GD, Bovet P, Shipley MJ, Marmot MG, Kumari M, et al. Association of lifecourse socioeconomic status with chronic inflammation and type 2 diabetes risk: the Whitehall II prospective cohort study. PLoS Med. 2013;10(7):e1001479.

27. Heraclides A, Witte D, Brunner EJ. The association between father's social class and adult obesity is not explained by educational attainment and an unhealthy lifestyle in adulthood. Eur J Epidemiol. 2008;23:579.
28. Brunner E, Shipley MJ, Blane D, Davey Smith G, Marmot MG. When does cardiovascular risk start? Past and present socioeconomic circumstances and risk factors in adulthood. J Epidemiol Community Health. 1999;53:764.

29. Blane D, Hart CL, Davey Smith G, Gillis CR, Hole DJ, Hawthorne VM. Association of cardiovascular disease risk factors with socioeconomic position during childhood and during adulthood. BMJ. 1996;313:1434-8.

30. Hart CL, Davey Smith G, Blane D. Social mobility and 21 year mortality in a cohort of Scottish men. Soc Sci Med. 1998:47:1121-30.

31. Popham F. Intergenerational social class stability and mobility are associated with large absolute differences in adult participation in sport and exercise. Br J Sports Med. 2010;44:1185.

32. Hart C, McConnachie A, Upton M, Watt G. Risk factors in the Midspan family study by social class in childhood and adulthood. Int J Epidemiol. 2008;37(3):604-14.

33. Silverwood RJ, Pierce M, Nitsch D, Mishra GD, Kuh D. Is intergenerational social mobility related to the type and amount of physical activity in mid-adulthood? Results from the 1946 British birth cohort study. Ann Epidemiol. 2012;22:487-98.

34. Kuh DJ, Cooper C. Physical activity at 36 years: patterns and childhood predictors in a longitudinal study. J Epidemiol Community Health. 1992;46:114-9.

35. Pinto Pereira SM, Li L, Power C. Early-life predictors of leisure-time physical inactivity in midadulthood: findings from a prospective British birth cohort. Am J Epidemiol. 2014;180(11):1098-108.

36. Cheng H, Furnham A. Personality traits, education, physical exercise, and childhood neurological function as independent predictors of adult obesity. PLoS One. 2013;8(11):e79586.

37. Juneau CE, Sullivan A, Dodgeon B, Côté S, Ploubidis GB, Potvin L. Social class across the life course and physical activity at age 34 years in the 1970 British birth cohort. Ann Epidemiol. 2014;24(9):641-7.

38. Osler M, Godtfredsen NS, Prescott E. Childhood social circumstances and health behaviour in midlife: the Metropolit 1953 Danish male birth cohort. Int J Epidemiol. 2008;37(6):1367-74.

39. Lynch JW, Kaplan GA, Salonen JT. Why do poor people behave poorly? Variation in adult health behaviours and psychosocial characteristics by stages of the socioeconomic lifecourse. Soc Sci Med. 1997;44(6):809-19.

40. Kvaavik E, Glymour M, Klepp KI, Tell GS, Batty GD. Parental education as a predictor of offspring behavioural and physiological cardiovascular disease risk factors. Eur J Public Health. 2012;22:550.

41. Jørgensen MB, Nabe-Nielsen K, Clausen T, Holtermann A. Independent effect of physical workload and childhood socioeconomic status on low back pain among health care workers in Denmark. Spine (Phila Pa 1976). 2013;38(6):E359-66.

42. Barnekow-Bergkvist M, Hedberg G, Janlert U, Jansson E. Prediction of physical fitness and physical activity level in adulthood by physical performance and physical activity in adolescence-an 18-year follow-up study. Scand J Med Sci Sports. 1998;8:299-308.

43. Tammelin T, Näyhä S, Laitinen J, Rintamäki H, Järvelin MR. Physical activity and social status in adolescence as predictors of physical inactivity in adulthood. Prev Med. 2003;37:375-81.

44. Makinen T, Kestila L, Borodulin K, Martelin T, Rahkonen O, Prattala R. Effects of childhood socio-economic conditions on educational differences in leisure-time physical activity. Eur J Public Health. 2010;20:353.

45. Wichstorm L, Kvalem IL, von Soest T. Predictors of growth and decline in leisure-time physical activity from adolescence to adulthood. Health Psychol. 2013;32(7):775-84.

46. Leino M, Raitakari OT, Porkka KV, Taimela S, Viikari JS. Associations of education with cardiovascular risk factors in young adults: the Cardiovascular Risk in Young Finns Study. Int J Epidemiol. 1999;28(4):667-75.

47. Osler M, Clausen JO, Ibsen KK, Jensen GB. Social influences and low leisure-time physical activity in young Danish adults. Eur J Public Health. 2001;11(2):130-4.

48. Peck MN. The importance of childhood socio-economic group for adult health. Soc Sci Med. 1994;39:553-62.

49. Regidor E, Banegas JR, Gutiérrez-Fisac JL, Domínguez V, Rodríguez-Artalejo F. Socioeconomic position in childhood and cardiovascular risk factors in older Spanish people. Int J Epidemiol. 2004;33(4):723-30.

50. Beunen GP, Lefevre J, Philippaerts RM, Delvaux K, Thomis M, Claessens AL, et al. Adolescent correlates of adult physical activity: a 26-year follow-up. Med Sci Sports Exerc. 2004;36(11):1930-6.

51. Scheerder J, Thomis M, Vanreusel B, Lefevre J, Renson R, Vanden Eynde B, et al. Sports participation among females from adolescence to adulthood: a longitudinal study. Int Rev Sociol Sport. 2006;41:413-30. 
52. Kamphuis CB, Turrell G, Giskes K, Mackenbach JP, van Lenthe FJ. Life course socioeconomic conditions, adulthood risk factors and cardiovascular mortality among men and women: a 17-year follow up of the GLOBE study. Int J Cardiol. 2013;168(3):2207-13.

53. Van de Mheen H, Stronks K, Looman CWN, Mackenbach JP. Does childhood socioeconomic status influence adult health through behavioural factors? Int J Epidemiol. 1998;27:437.

54. Pudrovska T, Anishkin A. Early-life socioeconomic status and physical activity in later life: evidence from structural equation models. J Aging Health. 2013:25:404

55. Wray LA, Alwin DF, McCammon RJ. Social status and risky health behaviors: results from the health and retirement study. J Gerontol B Psychol Sci Soc Sci. 2005;60:92.

56. Bowen ME. Coronary heart disease from a life-course approach: findings from the health and retirement study, 1998-2004. J Aging Health. 2010;22:241.

57. Carroll JE, Cohen S, Marsland AL. Early childhood socioeconomic status is associated with circulating interleukin-6 among mid-life adults. Brain Behav Immun. 2011;25:1474.

58. Frank $E$, Elon $L$, Hogue C. Transgenerational persistence of education as a health risk: findings from the women physicians' health study. J Women's Health. 2003;12:512.

59. Tsenkova V, Pudrovska T, Karlamangla A. Childhood socioeconomic disadvantage and prediabetes and diabetes in later life: a study of biopsychosocial pathways. Psychosom Med. 2014;76(8):622-8.

60. Kern ML, Reynolds CA, Friedman HS. Predictors of physical activity patterns across adulthood: a growth curve analysis. Pers Soc Psychol Bull. 2010;36(8):1058-72.

61. Phillips JE, Marsland AL, Flory JD, Muldoon MF, Cohen S, Manuck SB. Parental education is related to C-reactive protein among female middle aged community volunteers. Brain Behav Immun. 2009;23:683.

62. Schooling CM, Jiang CQ, Lam TH, Zhang WS, Cheng KK, Leung GM. Lifecourse origins of social inequalities in metabolic risk in the population of a developing country. Am J Epidemiol. 2008;167:419-28.

63. Elwell-Sutton TM, Jiang CQ, Zhang WS, Cheng KK, Lam TH, Leung GM, et al. Socioeconomic influences at different life stages on health in Guangzhou, China. Soc Sci Med. 2011;72:189.

64. Gall SL, Abbott-Chapman J, Patton GC, Dwyer T, Venn A. Intergenerational educational mobility is associated with cardiovascular disease risk behaviours in a cohort of young Australian adults: the Childhood Determinants of Adult Health (CDAH) Study. BMC Public Health. 2010;10:55.

65. Birnie K, Cooper R, Martin RM, Kuh D, Aihie Sayer A, Alvarado BE, et al. Childhood socioeconomic position and objectively measured physical capability levels in adulthood: a systematic review and meta-analysis. PLoS One. 2011;6(1):e15564.

66. Galobardes B, Davey Smith G, Lynch JW. Systematic review of the influence of childhood socioeconomic circumstances on risk for cardiovascular disease in adulthood. Ann Epidemiol. 2006;16(2):91-104.

67. Galobardes B, Lynch JW, Davey SG. Is the association between childhood socioeconomic circumstances and cause-specific mortality established? Update of a systematic review. J Epidemiol Community Health. 2008:62:387-90.

68. Kraaykamp G, Oldenkamp M, Breedveld K. Starting a sport in the Netherlands: a life-course analysis of the effects of individual, parental and partner characteristics. Int Rev Sociol Sport. 2013;48:153-70.

69. Graham H. Socio-economic inequalities across generations: occupation and education. In: Unequal lives: health and socioeconomic inequalities. Berkshire: Open University Press; 2007. p. 114-32.

70. Telama R. Tracking of physical activity from childhood to adulthood: a review. Obes Facts. 2009;2(3):187-95.

71. Yao CA, Rhodes RE. Parental correlates in child and adolescent physical activity: a meta-analysis. Int J Behav Nutr Phys Act. 2015;12:10.

72. Schooling M, Kuh D. A life course perspective on women's health behaviours. In: Kuh D, Hardy R, editors. A life course approach to women's health. Oxford: Oxford University Press; 2003. p. 279-303.

73. Malina RM, Bouchard C, Bar-Or O. Other factors affecting growth, maturation, performance, and activity. In: Malina RM, Bouchard C, Bar-Or O, editors. Growth, maturation, and physical activity. 2nd ed. United States: Human Kinetics; 2004. p. 553-77.

74. Aaltonen S, Ortega-Alonso A, Kujala UM, Kaprio J. Genetic and environmental influences on longitudinal changes in leisure-time physical activity from adolescence to young adulthood. Twin Res Hum Genet. 2013;16(2):535-43.
75. Bauman AE, Reis RS, Sallis JF, Wells JC, Loos RJ, Martin BW. Lancet Physical Activity Series Working Group: correlates of physical activity: why are some people physically active and others not? Lancet. 2012;380(9838):258-71.

76. Jones RA, Hinkley T, Okely AD, Salmon J. Tracking physical activity and sedentary behavior in childhood: a systematic review. Am J Prev Med. 2013;44(6):651-8.

77. Batty GD, Lawlor DA, Macintyre S, Clark H, Leon DA. Accuracy of adults' recall of childhood social class: findings from the Aberdeen children of the 1950s study. J Epidemiol Community Health. 2005;59(10):898-903.

78. Bann D, Cooper R, Wills AK, Adams J, Kuh D, on behalf of NSHD scientific and data collection team. Socioeconomic position across life and body composition in early old age: findings from a British birth cohort study. J Epidemiol Community Health. 2014;68(6):516-23.

79. Mackenbach JP, Stirbu I, Roskam AJ, Schaap MM, Menvielle G, Leinsalu M, et al. Socioeconomic inequalities in health in 22 European countries. N Engl J Med. 2008;358(23):2468-81.

80. Wilcox S, Castro C, King AC, Housemann R, Brownson RC. Determinants of leisure time physical activity in rural compared with urban older and ethnically diverse women in the United States. J Epidemiol Community Health. 2000;54(9):667-72.

81. Kim S, Symons M, Popkin BM. Contrasting socioeconomic profiles related to healthier lifestyles in China and the United States. Am J Epidemiol. 2004;159(2):184-91.

82. Golubic R, Martin KR, Ekelund U, Hardy R, Kuh D, Wareham N, et al. Levels of physical activity among a nationally representative sample of people in early old age: results of objective and self-reported assessments. Int J Behav Nutr Phys Act. 2014;11:58.

\section{Submit your next manuscript to BioMed Central and take full advantage of:}

- Convenient online submission

- Thorough peer review

- No space constraints or color figure charges

- Immediate publication on acceptance

- Inclusion in PubMed, CAS, Scopus and Google Scholar

- Research which is freely available for redistribution 